\title{
Congruence Subgroups Associated to the Monster
}

\author{
Kok Seng Chua and Mong Lung Lang
}

\section{CONTENTS}

1. Introduction

2. Genus Formula

3. The Finiteness of $\Delta$

4. The Determination of $\Delta$

5. Elliptic Periods

6. The Cusps of $\Gamma_{0}(N)$ and $\Gamma(N)$

7. Proof

References

2000 AMS Subject Classification: Primary 20H05, 11F06

Keywords: Congruence subgroups, genus, Monster simple group
Let $\Delta=\left\{G: g(G)=0, \Gamma_{0}(m) \leq G \leq N\left(\Gamma_{0}(m)\right)\right.$ for some $m\}$, where $N\left(\Gamma_{0}(m)\right)$ is the normaliser of $\Gamma_{0}(m)$ in $P S L_{2}(\mathbb{R})$ and $g(G)$ is the genus of $\mathbb{H}^{*} / G$. In this article, we determine all the $m$. Further, for each $m$, we list all the intermediate groups $G$ of $\Gamma_{0}(m) \leq N\left(\Gamma_{0}(m)\right)$ such that $g(G)=0$. All the intermediate groups of width 1 at $\infty$ are also listed in a separate table (see www.math.nus.edu.sg/ matlml/).

\section{INTRODUCTION}

Let $m \in \mathbb{N}$ and let $h$ be the largest divisor of 24 such that $m=n h^{2}$. The normaliser of $\Gamma_{0}(m)$ is given by

$N\left(\Gamma_{0}(m)\right)=\Gamma_{0}^{+}(n h \mid h)=\left(\begin{array}{cc}h & 0 \\ 0 & 1\end{array}\right)^{-1} \Gamma_{0}^{+}(n)\left(\begin{array}{cc}h & 0 \\ 0 & 1\end{array}\right)$,

where $\Gamma_{0}^{+}(n)$ is the group generated by $\Gamma_{0}(n)$ and all the Atkin-Lehner involutions associated to $\Gamma_{0}(n)$ (see [Atkin and Lehner 70, Akbas and Singerman 90, Conway 79, Conway 96]). Recall that for each exact divisor $e$ of $n$ ( $e$ is an exact divisor of $n$ if $\operatorname{gcd}(e, n / e)=1$ ), an AtkinLehner involution $w_{e}$ associated to $\Gamma_{0}(n)$ is of the form

$$
w_{e}=\left(\begin{array}{cc}
a \sqrt{e} & b / \sqrt{e} \\
c n / \sqrt{e} & d \sqrt{e}
\end{array}\right)
$$

In [Conway 79], Conway and Norton raised the question: which groups between $\Gamma_{0}(m)$ and its normaliser have genus zero? The purpose of this article is to give an answer to this question. Define

$$
\begin{gathered}
\Delta=\left\{G: g(G)=0, \Gamma_{0}(m) \leq G \leq N\left(\Gamma_{0}(m)\right)\right. \\
\text { for some } m\} \\
\Omega=\left\{m: g\left(N\left(\Gamma_{0}(m)\right)\right)=0\right\}
\end{gathered}
$$

It is clear that

$$
\Delta=\left\{G: g(G)=0, \Gamma_{0}(m) \leq G \leq N\left(\Gamma_{0}(m)\right), m \in \Omega\right\}
$$

Our main results include (i) the determination of $\Omega$, and (ii) the determination of the set of all intermediate groups 
$G$ of $\Gamma_{0}(m) \leq N\left(\Gamma_{0}(m)\right)$ of genus zero (see Section 4.1). This answers the question of Conway and Norton. Note that groups in $\Delta$ have relevance for the Monster simple group (see [Conway 79, Thompson 80] for examples) and such groups of $n \mid h$-type have been determined by C. R. Ferenbaugh [Ferenbaugh 93]. The set $\Delta$ can be found on our web site. As for the set $\Omega$, we may write each $m$ in $\Omega$ into $m=n h^{2}$, where $h$ is the largest divisor of 24 such that $h^{2} \mid m$. Then the pair $(n, h)$ can be found in Table 1 (see Section 4.2). There are 419 of them. In particular, the $n$ ( 64 of them) can be found in the following: 1, 2, 3, $4,5,6,7,8,9,10,11,12,13,14,15,16,17,18,19,20$, $21,22,23,24,25,26,27,29,30,31,32,33,34,35,36$, $38,39,41,42,44,45,46,47,49,50,51,54,55,56,59,60$, $62,66,69,70,71,78,87,92,94,95,105,110,119$. Table 3 also provides us with the number of conjugacy classes, the numbers of intermediate groups, and the number of groups having width 1 at infinity. Congruence subgroups of $P S L_{2}(\mathbb{Z})$ of genus up to 24 have been determined by $\mathrm{C}$. Cummins and S. Pauli [Cummins and Pauli 03]. Torsionfree genus zero congruence subgroups of $P S L_{2}(\mathbb{R})$ have been determined by A. Sebbar [Sebbar 01].

The main fact used in our study is a simple theorem that determines the genera of all the intermediate groups of $\Gamma_{0}(m) \leq N\left(\Gamma_{0}(m)\right)$ (see Theorem 2.5).

The rest of this article is organised as follows: in Section 2, we determine the genus formula of $G$, where $G$ is an intermediate group of $\Gamma_{0}(m) \leq N\left(\Gamma_{0}(m)\right)$, for any $m$. In particular, this formula can be implemented using the software package GAP (Groups, Algorithms, and Programming). Section 3 proves that $\Omega$ and $\Delta$ are finite. Section 4 provides us with a list of some intermediate groups of $\Gamma_{0}(16) \leq N\left(\Gamma_{0}(16)\right)$ of genus 0 and a web site (www.math.nus.edu.sg/ $\sim$ matlml/) that gives the set $\Delta$ and all the subgroups in $\Delta$ of width 1 (at $\infty$ ). Table 4 gives the signature of $\Gamma_{0}^{+}(n)$, where $n \in E$ (see Section 3 for the definition of $E$ ). Table 5 gives all the intermediate groups $G$ of $\Gamma_{0}(n) \leq \Gamma_{0}^{+}(n)$ such that $g(G)=0$. Section 5 gives a set of representatives of nonconjugate elliptic subgroups of orders $2,3,4,6$ of $\Gamma_{0}^{+}(n), n \in E$. Section 6 gives a systematic description of cusps of $\Gamma_{0}\left(n h^{2}\right)$ and $\Gamma(n)$. The permutation representations of $\Gamma_{0}^{+}(n h \mid h)$ and $P S L_{2}(\mathbb{Z})$ on the sets of cusps of $\Gamma_{0}\left(n h^{2}\right)$ and $\Gamma(n)$, respectively, are also determined in Section 6 .

It is our pleasure and duty to report that congruence subgroups of $P S L_{2}(\mathbb{R})$ of genus 0 and 1 have been determined by Cummins [Cummins 04]. This is achieved by studying the quotient groups $\Gamma_{0}^{+}(f) / \Gamma_{0}(n f) \cap \Gamma(n)$, where $f$ is square-free. It is clear that every group in our list (www.math.nus.edu.sg/ matlml/) must conjugate to one of the groups listed in Table 3 of Cummins. However, it is not an easy task to compare these two lists as we are working in different quotient groups $\left(\Gamma_{0}^{+}(f) / \Gamma_{0}(n f) \cap\right.$ $\Gamma(n)$ for Cummins and $N\left(\Gamma_{0}(m)\right) / \Gamma_{0}(m)$ for Chua and Lang) and the groups are presented differently (invariants of the groups for Cummins and matrices modulo $\Gamma_{0}\left(n h^{2}\right)$ for Chua and Lang). Comparison is possible only when groups admit the following properties:

(i) groups of Chua and Lang of width 1 (at $\infty$ ),

(ii) groups of Cummins that normalise $\Gamma_{0}(m)$ for some $m$.

The result of this comparison is given in Table 7 of Cummins and Table 3 of Chua and Lang separately and is in agreement. To be more precise, let $\left(c_{1}, c_{2}, c_{3}\right)$ (of Cummins) and $\left(c l_{1}, c l_{2}, c l_{3}\right)$ (of Chua and Lang) be the corresponding triples, then

$$
c=c_{3}=c l_{3},
$$

where $c$ is the number of intermediate groups of genus 0 , width $1($ at $\infty)$ of $\Gamma_{0}\left(n h^{2}\right) \leq N\left(\Gamma_{0}\left(n h^{2}\right)\right)=\Gamma_{0}^{+}(n h \mid h)$.

\section{GENUS FORMULA}

The main purpose of this section is to give a genus formula of intermediate groups of $\Gamma_{0}(m) \leq N\left(\Gamma_{0}(m)\right)$ that can be implemented in GAP. Recall first that if $G$ is a subgroup of $P S L_{2}(\mathbb{R})$ commensurable with $P S L_{2}(\mathbb{Z})$, then

$$
\chi(G)=2(g(G)-1)+c+\sum_{i=1}^{r}\left(1-1 / d_{i}\right),
$$

where $-\chi(G)$ is the Euler characteristic, $c$ is the number of cusps of $G, r$ is the number of nonconjugating elliptic subgroups of $G$, and $d_{1}, d_{2}, \cdots, d_{r}$ are their orders.

Recall that $\langle\sigma\rangle$ is an elliptic subgroup of $\Gamma$ if $\langle\sigma\rangle$ is a maximal cyclic subgroup of $\Gamma$. An element $e$ is called an elliptic element if $\langle e\rangle$ is an elliptic subgroup.

Remark 2.1. Let $m \in \mathbb{N}$. Then $\chi\left(\Gamma_{0}^{+}(m)\right)=\frac{m}{6} \prod_{p \mid m}(p+$ 1) $/ 2 p$, where $p$ runs through all the prime divisors of $m$.

Let $G$ be an intermediate group of $\Gamma_{0}(m)=\Gamma_{0}\left(n h^{2}\right) \leq$ $N(\Gamma(m))=\Gamma_{0}^{+}(n h \mid h)$. Applying $(2-1)$, the genus $g(G)$ satisfies

$$
\begin{aligned}
\chi\left(\Gamma_{0}^{+}(n)\right)\left[\Gamma_{0}^{+}(n h \mid h): G\right]= & 2(g(G)-1)+c+\frac{5 v_{6}}{6}+\frac{3 v_{4}}{4} \\
& +\frac{2 v_{3}}{3}+\frac{v_{2}}{2},
\end{aligned}
$$


where $c$ is the number of cusps of $G$ and $v_{n}=v_{n}(G)$ is the number of nonconjugating elliptic subgroups of order $n$ of $G$. The set $\left\{g(G), c, v_{2}, v_{3}, v_{4}, v_{6}\right\}$ is called the signature of $G$.

\subsection{Signature of $\Gamma_{0}^{+}(n)$, Where $n$ Is Square-Free}

The purpose of this section is to determine the signature of $\Gamma_{0}^{+}(n)$, where $n$ is square-free. Recall first that since $n$ is square-free, $\Gamma_{0}^{+}(n)$ has a unique cusp. Applying results of Maclachlan [Maclachlan 81] and Akbas and Singerman [Akbas and Singerman 92, Theorem 2], we have:

Theorem 2.2. Let $n$ be an integer (not necessarily squarefree). Then $v_{6}\left(\Gamma_{0}^{+}(n)\right), v_{3}\left(\Gamma_{0}^{+}(n)\right)$, and $v_{4}\left(\Gamma_{0}^{+}(n)\right)$ are either 1 or 0 and

(a) $v_{6}\left(\Gamma_{0}^{+}(n)\right)=1$ iff $3 \mid n$ and all the divisors of $n / 3$ are of the form $3 k+1$,

(b) $v_{4}\left(\Gamma_{0}^{+}(n)\right)=1$ iff $2 \mid n$ and all the divisors of $n / 2$ are of the form $4 k+1$,

(c) $v_{3}\left(\Gamma_{0}^{+}(n)\right)=1$ iff all the divisors of $n$ are of the form $3 k+1$.

Applying Theorem 2.2, representatives of nonconjugate elliptic subgroups of orders 3,4 , and 6 can be obtained easily (see Section 5). Since $n$ is square-free, $v_{2}\left(\Gamma_{0}^{+}(n)\right)$ is given by Maclachlan [Maclachlan 81]. The determination of representatives of nonconjugate elliptic subgroups of order 2 of $\Gamma_{0}^{+}(n)$ can be reduced to a simple study of certain positive definite quadratic forms. In particular, a complete list of representatives of nonconjugate elliptic subgroups of order 2 of $\Gamma_{0}^{+}(n)$, where $g\left(\Gamma_{0}^{+}(n)\right)=0$, can be found in Section 5 . Note that Theorem 2.2 implies that

$$
\begin{aligned}
v_{6}\left(\Gamma_{0}^{+}(n)\right) \cdot v_{4}\left(\Gamma_{0}^{+}(n)\right) & =v_{6}\left(\Gamma_{0}^{+}(n)\right) \cdot v_{3}\left(\Gamma_{0}^{+}(n)\right) \\
& =v_{3}\left(\Gamma_{0}^{+}(n)\right) \cdot v_{4}\left(\Gamma_{0}^{+}(n)\right) \\
& =0 .
\end{aligned}
$$

\subsection{Signature of $\Gamma_{0}^{+}(n)$, Where $n$ Is Not Necessarily Square-Free}

The purpose of this section is to determine the signature of $\Gamma_{0}^{+}(n)$ for any $n$. Note first that $v_{k}\left(\Gamma_{0}^{+}(n)\right), k \geq 3$, can be determined by Theorem 2.2. As a consequence, representatives of nonconjugate elliptic subgroups of orders 3 , 4 , and 6 can be obtained easily. The number of cusps of $\Gamma_{0}^{+}(n)$ can be determined by applying results of [Akbas and Singerman 92]. In order to determine a set of nonconjugate elliptic subgroups of 2 , we recall the following results of [Lang 01, Section 4]. For readers' convenience, a proof of (i) of the following can be found in Section 7 .

Suppose that $G$ is a subgroup of $\Gamma_{0}^{+}(f B \mid B)$. Let $\Gamma_{0}^{+}(f B \mid B)=\cup g_{i} G$ and let $\left\{\tau_{1}, \tau_{2}, \cdots, \tau_{s}\right\}$ be a complete set of representatives of nonconjugate elliptic subgroups of order 2 of $\Gamma_{0}^{+}(f B \mid B)$.

(i) If $v_{6}\left(\Gamma_{0}^{+}(f B \mid B)\right)=1$, then $v_{4}\left(\Gamma_{0}^{+}(f B \mid B)\right)=$ $v_{3}\left(\Gamma_{0}^{+}(f B \mid B)\right)=0$. Let $u$ be an element of order 6 of $\left.\Gamma_{0}^{+}(f B \mid B)\right)$. Suppose that $\mid\left\{g_{i}: g_{i}^{-1} u g_{i} \in\right.$ $G\}|=r|,\left\{g_{i}: g_{i}^{-1} u^{2} g_{i} \in G\right\}|=k|,\left\{g_{i}:\right.$ $\left.g_{i}^{-1} \tau_{j} g_{i} \in G\right\}\left|=e_{j},\right|\left\{g_{i}: g_{i}^{-1} u^{3} g_{i} \in G\right\} \mid=e$. Then $v_{4}(G)=0, v_{6}(G)=r, v_{3}(G)=(k-r) / 2$, $v_{2}(G)=e_{1}+e_{2}+\cdots+e_{s}+(e-r) / 3$.

(ii) If $v_{4}\left(\Gamma_{0}^{+}(f B \mid B)\right)=1$, then $v_{3}\left(\Gamma_{0}^{+}(f B \mid B)\right)=$ $v_{6}\left(\Gamma_{0}^{+}(f B \mid B)\right)=0$. Let $u$ be an element of order 4 of $\left.\Gamma_{0}^{+}(n h \mid h)\right)$. Suppose that $\left|\left\{g_{i}: g_{i}^{-1} u g_{i} \in G\right\}\right|=r$, $\left|\left\{g_{i}: g_{i}^{-1} u^{2} g_{i} \in G\right\}\right|=k,\left|\left\{g_{i}: g_{i}^{-1} \tau_{j} g_{i} \in G\right\}\right|=$ $e_{j}$. Then $v_{4}(G)=r, v_{6}(G)=v_{3}(G)=0$, and $v_{2}(G)=e_{1}+e_{2}+\cdots+e_{s}+(k-r) / 2$.

(iii) If $v_{3}\left(\Gamma_{0}^{+}(f B \mid B)\right)=1$, then $v_{4}\left(\Gamma_{0}^{+}(f B \mid B)\right)=$ $v_{6}\left(\Gamma_{0}^{+}(f B \mid B)\right)=0$. Let $u$ be an element of order 3 of $\Gamma_{0}^{+}(f B \mid B)$. Suppose that $\left|\left\{g_{i}: g_{i}^{-1} u g_{i} \in G\right\}\right|=r$, $\left|\left\{g_{i}: g_{i}^{-1} \tau_{j} g_{i} \in G\right\}\right|=e_{j}$. Then $v_{4}(G)=v_{6}(G)=$ $0, v_{3}(G)=r, v_{2}(G)=e_{1}+e_{2}+\cdots+e_{s}$.

(iv) Suppose that $v_{3}\left(\Gamma_{0}^{+}(f B \mid B)\right)=v_{4}\left(\Gamma_{0}^{+}(f B \mid B)\right)=$ $v_{6}\left(\Gamma_{0}^{+}(f B \mid B)\right)=0$. Suppose further that $\mid\left\{g_{i}\right.$ : $\left.g_{i}^{-1} \tau_{j} g_{i} \in G\right\} \mid=e_{j}$. Then $v_{4}(G)=v_{6}(G)=v_{3}(G)=$ $0, v_{2}(G)=e_{1}+e_{2}+\cdots+e_{s}$.

Let $G=\Gamma_{0}^{+}(n)$ and let $n=f B^{2}$, where $f$ is squarefree. Since $f$ is square-free, a complete list of representatives of nonconjugate elliptic subgroups of order 2 of $\Gamma_{0}^{+}(f B \mid B)$ can be determined by our results in Section 5. Applying the above results, the number of nonconjugating elliptic subgroups of order 2 of $\Gamma_{0}^{+}(n)$ can be determined. The set of representatives of nonconjugating elliptic subgroups of order 2 of $\Gamma_{0}^{+}(n)$ can now be determined by applying our results in Section 5 .

\subsection{Genus of Intermediate Groups of $\Gamma_{0}(m) \leq N\left(\Gamma_{0}(m)\right)$}

Throughout the section, $m=n h^{2}$, where $h$ is the largest divisor of 24 such that $h^{2} \mid m$. Since $\Gamma_{0}(m)$ is a normal subgroup of $N\left(\Gamma_{0}(m)\right)=\Gamma_{0}^{+}(n h \mid h), \Gamma_{0}^{+}(n h \mid h)$ acts on the set of cusps of $\Gamma_{0}(m)$. Denote the action by $\rho$.

Lemma 2.3. Let $K=\Gamma_{0}^{+}(n h \mid h)$. Then $\rho(K) \cong$ $K / \Gamma_{0}\left(n h^{2}\right)$. 
Proof: Suppose not. Let $\sigma \in K-\Gamma_{0}\left(n h^{2}\right)$ be chosen such that $\rho(\sigma)=1$. This implies that $\rho(\sigma)$ fixes the cusp $[\infty]$. It follows that $\sigma(\infty)=\tau(\infty)$ for some $\tau \in \Gamma_{0}\left(n h^{2}\right)$. Hence

$$
\tau^{-1} \sigma=\left(\begin{array}{cc}
1 & x / h \\
0 & 1
\end{array}\right)
$$

for some $x \in \mathbb{Z}$. Since $\tau^{-1} \sigma \in K-\Gamma_{0}\left(n h^{2}\right), x$ is not a multiple of $h$. As a consequence, $\tau^{-1} \sigma$ does not fix the cusp [0] $=\left\{g(0): g \in \Gamma_{0}\left(n h^{2}\right)\right\}=\left\{x / y: \operatorname{gcd}\left(y, n h^{2}\right)=\right.$ 1\}. As $\tau \in \Gamma_{0}\left(n h^{2}\right)$, we conclude that $\sigma$ does not fix [0]. Hence $\rho(\sigma) \neq 1$. A contradiction. Hence $\rho(K) \cong$ $K / \Gamma_{0}\left(n h^{2}\right)$.

Lemma 2.4. Let $A \leq B$ be finite groups and let $\left\{b_{1}, b_{2}, \cdots, b_{k}\right\}$ be a set of left coset representatives of $A$ in $B$. Let $g \in B$. Then

$$
\left|\left\{b_{i}: b_{i}^{-1} g b_{i} \in A\right\}\right|=[B: A]\left|C l_{B}(g) \cap A\right| /\left|C l_{B}(g)\right| .
$$

Proof: Let $\Delta=\left\{b_{i}: b_{i}^{-1} g b_{i} \in A\right\}$. Suppose that $m=$ $\left|\left\{b_{i}^{-1} g b_{i}: b_{i} \in \Delta\right\}\right|$. For our convenience, we may assume that $\left\{b_{i}^{-1} g b_{i}: b_{i} \in \Delta\right\}=\left\{b_{i}^{-1} g b_{i}: i=1,2, \cdots, m\right\}$. It is clear that

$$
\Delta=\cup_{i=1}^{m}\left\{b_{i_{n}}: b_{i_{n}}^{-1} g b_{i_{n}}=b_{i}^{-1} g b_{i}\right\} \text { (disjoint union). }
$$

Let $\Psi=\left\{b_{i}^{-1} g b_{i}: i=1,2, \cdots, m\right\}$. For each $b^{-1} g b \in$ $\Psi$, it is clear that

$$
\begin{gathered}
b_{i}^{-1} g b_{i}=b^{-1} g b \text { iff } b_{i}^{-1} b b^{-1} g b b^{-1} b_{i}=b^{-1} g b \\
\text { iff } b^{-1} b_{i} \in C_{B}\left(b^{-1} g b\right)=\cup_{t=1}^{s} x_{t} C_{A}\left(b^{-1} g b\right) .
\end{gathered}
$$

One can now prove that there are exactly $\left|C_{B}\left(b^{-1} g b\right) / C_{A}\left(b^{-1} g b\right)\right|$ choices for $b_{i}$ such that $(2-5)$ holds. It follows that

$$
\left\{b_{i_{n}}: b_{i_{n}}^{-1} g b_{i_{n}}=b_{i}^{-1} g b_{i}\right\}=\left|C_{B}\left(b_{i}^{-1} g b_{i}\right) / C_{A}\left(b_{i}^{-1} g b_{i}\right)\right| .
$$

Applying (2-4) and (2-6), we have

$$
\begin{aligned}
|\Delta| & =\sum_{i=1}^{m}\left|C_{B}\left(b_{i}^{-1} g b_{i}\right) / C_{A}\left(b_{i}^{-1} g b_{i}\right)\right| \\
& =[B: A]\left|C l_{B}(g) \cap A\right| /\left|C l_{B}(g)\right| .
\end{aligned}
$$

This completes the proof of the lemma.

By Lemma 2.3, there is a one-to-one correspondence between the intermediate groups of $\Gamma_{0}\left(n h^{2}\right) \leq \Gamma_{0}^{+}(n h \mid h)$ and subgroups of $\rho\left(\Gamma_{0}^{+}(n h \mid h)\right)$. As a consequence, (i), (ii), (iii), and (iv) of Section 2.2 and the genus formula
(2-2) can be evaluated in the finite group $\rho\left(\Gamma_{0}^{+}(n h \mid h)\right)$. By Lemma 2.4, we have the following:

Theorem 2.5. Let $\left\{\tau_{1}, \tau_{2}, \cdots, \tau_{s}\right\}$ be a complete set of representatives of nonconjugate elliptic subgroups of order 2 of $\Gamma_{0}^{+}(n h \mid h)$ and let $G$ be an intermediate group of $\Gamma_{0}(m) \leq N\left(\Gamma_{0}(m)\right)=\Gamma_{0}^{+}(n h \mid h)$. Then $G$ possesses $c$ cusps, where $c$ is the number of $\rho(G)$ orbits.

$$
\begin{aligned}
g(G)= & 1+\chi\left(\Gamma_{0}^{+}(n)\right)\left[\Gamma_{0}^{+}(n h \mid h): G\right] / 2-c / 2 \\
& -\frac{5 v_{6}(G)}{12}-\frac{3 v_{4}(G)}{8}-\frac{2 v_{3}(G)}{6}-\frac{v_{2}(G)}{4},
\end{aligned}
$$

where $-\chi\left(\Gamma_{0}^{+}(n)\right)$ is the Euler characteristic. Further, $v_{n}(G)$ is given as follows:

(i) Suppose that $v_{6}\left(\Gamma_{0}^{+}(n h \mid h)\right)=1$. Then

$$
v_{3}\left(\Gamma_{0}^{+}(n h \mid h)\right)=v_{4}\left(\Gamma_{0}^{+}(n h \mid h)\right)=0 .
$$

Let $u$ be an element of $\Gamma_{0}^{+}(n h \mid h)$ of order 6 . Denote by $\mathrm{Cl}(u)$ the conjugacy class of $u$ in $\rho\left(\Gamma_{0}^{+}(n h \mid h)\right.$, then

$$
\begin{aligned}
r & =\left[\Gamma_{0}^{+}(n h \mid h): G\right]|C l(\rho(u)) \cap \rho(G)| /|C l(\rho(u))|, \\
k & =\left[\Gamma_{0}^{+}(n h \mid h): G\right]|| C l\left(\rho\left(u^{2}\right)\right) \cap \rho(G)|/| C l\left(\rho\left(u^{2}\right)\right), \\
e_{j} & =\left[\Gamma_{0}^{+}(n h \mid h): G\right]\left|C l\left(\rho\left(\tau_{j}\right)\right) \cap \rho(G)\right| / \mid C l\left(\rho\left(\tau_{j}\right)\right), \\
e & =\left[\Gamma_{0}^{+}(n h \mid h): G\right]\left|C l\left(\rho\left(u^{3}\right)\right) \cap \rho(G)\right| /\left|C l\left(\rho\left(u^{3}\right)\right)\right|, \\
v_{4}(G) & =0, \\
v_{6}(G) & =r, \\
v_{3}(G) & =(k-r) / 2, \\
v_{2}(G) & =e_{1}+e_{2}+\cdots+e_{s}+(e-r) / 3 .
\end{aligned}
$$$$
v_{4}(G)=0 \text {, }
$$$$
v_{6}(G)=r
$$

(ii) Suppose that $v_{4}\left(\Gamma_{0}^{+}(n h \mid h)\right)=1$. Then

$$
v_{3}\left(\Gamma_{0}^{+}(n h \mid h)\right)=v_{6}\left(\Gamma_{0}^{+}(n h \mid h)\right)=0 .
$$

Let $u$ be an element of $\Gamma_{0}^{+}(n h \mid h)$ of order 4 . Then

$$
\begin{aligned}
r= & {\left[\Gamma_{0}^{+}(n h \mid h): G\right]|C l(\rho(u)) \cap \rho(G)| /|C l(\rho(u))|, } \\
k & =\left[\Gamma_{0}^{+}(n h \mid h): G\right]|| C l\left(\rho\left(u^{2}\right)\right) \cap \rho(G)|/| C l\left(\rho\left(u^{2}\right)\right), \\
e_{j} & =\left[\Gamma_{0}^{+}(n h \mid h): G\right]\left|C l\left(\rho\left(\tau_{j}\right)\right) \cap \rho(G)\right| / \mid C l\left(\rho\left(\tau_{j}\right)\right), \\
v_{4}(G) & =r \\
v_{6}(G) & =v_{3}(G)=0, \\
v_{2}(G) & =e_{1}+e_{2}+\cdots+e_{s}+(k-r) / 2 .
\end{aligned}
$$

(iii) Suppose that $v_{3}\left(\Gamma_{0}^{+}(n h \mid h)\right)=1$. Then

$$
v_{4}\left(\Gamma_{0}^{+}(n h \mid h)\right)=v_{6}\left(\Gamma_{0}^{+}(n h \mid h)\right)=0 .
$$


Let $u$ be an element of $\Gamma_{0}^{+}(n h \mid h)$ of order 4 . Then

$$
\begin{aligned}
r & =\left[\Gamma_{0}^{+}(n h \mid h): G\right]|C l(\rho(u)) \cap \rho(G)| /|C l(\rho(u))|, \\
e_{j} & =\left[\Gamma_{0}^{+}(n h \mid h): G\right]\left|C l\left(\rho\left(\tau_{j}\right)\right) \cap \rho(G)\right| / \mid C l\left(\rho\left(\tau_{j}\right)\right), \\
v_{3}(G) & =r \\
v_{6}(G) & =v_{4}(G)=0 \\
v_{2}(G) & =e_{1}+e_{2}+\cdots+e_{s} .
\end{aligned}
$$

(iv) Suppose that

$$
v_{3}\left(\Gamma_{0}^{+}(n h \mid h)\right)=v_{4}\left(\Gamma_{0}^{+}(n h \mid h)\right)=v_{6}\left(\Gamma_{0}^{+}(n h \mid h)\right)=0 .
$$

Then

$$
\begin{aligned}
e_{j} & =\left[\Gamma_{0}^{+}(n h \mid h): G\right]\left|C l\left(\rho\left(\tau_{j}\right)\right) \cap \rho(G)\right| / \mid C l\left(\rho\left(\tau_{j}\right)\right), \\
v_{3}(G) & =v_{6}(G)=v_{4}(G)=0 \\
v_{2}(G) & =e_{1}+e_{2}+\cdots+e_{s} .
\end{aligned}
$$

\section{THE FINITENESS OF $\boldsymbol{\Delta}$}

Recall first that

$$
\begin{aligned}
& \Delta=\left\{G: g(G)=0, \Gamma_{0}(m) \leq G \leq N\left(\Gamma_{0}(m)\right)\right. \\
& \quad \text { for some } m\}, \\
& \Omega=\left\{m: g\left(N\left(\Gamma_{0}(m)\right)\right)=g\left(\Gamma_{0}^{+}(n h \mid h)\right)=0\right\} .
\end{aligned}
$$

The main purpose of this section is to show that $\Omega$ and $\Delta$ are finite (see Sections 3.1 and 3.2). In order to achieve this, we define the following set:

$$
E=\left\{n: g\left(\Gamma_{0}^{+}(n)\right)=0\right\} .
$$

We use the following results by Zograf [Zograf 91].

Theorem 3.1. [Zograf 91] Let $\Gamma$ be a subgroup of $P S L_{2}(\mathbb{R})$ commensurable with $P S L_{2}(\mathbb{Z})$ and let $G$ be a congruence subgroup of $\Gamma$. Then $g(G)+1>3 \chi(\Gamma)[\Gamma: G] / 64$, where $-\chi(\Gamma)$ is the Euler characteristic,

$$
\chi(\Gamma)=2(g(\Gamma)-1)+c+\sum_{i=1}^{r}\left(1-1 / d_{i}\right),
$$

$c$ is the number of cusps of $\Gamma, r$ is the number of conjugacy classes of elliptic subgroups of $\Gamma$, and $d_{1}, d_{2}, \cdots, d_{r}$ are their orders.

Corollary 3.2. Let $g_{0} \in \mathbb{N}$ and let $E_{g_{0}}=\left\{n: g\left(\Gamma_{0}^{+}(n)\right) \leq\right.$ $\left.g_{0}\right\}$. Then $E_{g_{0}}$ is finite. Further, if $n \in E_{g_{0}}$. Then

$$
128\left(g_{0}+1\right) \geq n \prod_{p \mid n}(p+1) / 2 p
$$

\subsection{The Sets $E$ and $\Omega$ Are Finite}

The main purpose of this section is to determine the sets $E=\left\{n: g\left(\Gamma_{0}^{+}(n)\right)=0\right\}$ and $\Omega$. Suppose that $g\left(\Gamma_{0}^{+}(f)\right)=0$, where $f$ is square-free. Let $\Gamma=G=$ $\Gamma_{0}^{+}(f)$; by Theorem 3.1, we have

$$
128>\prod_{p \mid f}(p+1) / 2 .
$$

Hence the possible prime divisors of $f$ are $2,3,5,7, \cdots, 251$. Direct calculation shows that a complete list of the $f$ such that $g\left(\Gamma_{0}^{+}(f)\right)=0$ is given by the following: $1,2,3,5,6,7,10,11,13,14,15,17,19,21,22,23,26,29,30$, $31,33,34,35,38,39,41,42,46,47,51,55,59,62,66,69,70$, $71,78,87,94,95,105,110,119$. Denote the above set by $F$.

Remark 3.3. Let $f \in F$. An easy observation shows that the possible prime divisors of $f$ are members of $\mathbb{M}_{p}=\{2,3,5,7,11,13,17,19,23,29,31,41,47,59,71\}$. Note that $\mathbb{M}_{p}$ is the set of prime divisors of the order of the Monster simple group.

Lemma 3.4. Let $E=\left\{n: g\left(\Gamma_{0}^{+}(n)\right)=0\right\}$. Then

$$
\begin{gathered}
E=F \cup\{4,8,9,12,16,18,20,24,25,27,32,36,44,45,49, \\
50,54,56,60,92\} .
\end{gathered}
$$

Proof: Suppose that $n \in E$. Let $n=f B^{2}$ ( $f$ is squarefree). Then

$$
\Gamma_{0}^{+}(n) \leq \Gamma_{0}^{+}(f B \mid B)=\left(\begin{array}{cc}
B & 0 \\
0 & 1
\end{array}\right)^{-1} \Gamma_{0}^{+}(f)\left(\begin{array}{cc}
B & 0 \\
0 & 1
\end{array}\right) .
$$

Since $\Gamma_{0}^{+}(n)$ is of genus 0 and $\Gamma_{0}^{+}(n)$ is a subgroup of $\left.\Gamma_{0}^{+}(f B \mid B)\right)$, we have $g\left(\Gamma_{0}^{+}(f)\right)=g\left(\Gamma_{0}^{+}(f B \mid B)\right)=0$. Hence $f \in F$. Let $\left.\Gamma=\Gamma_{0}^{+}(f B \mid B)\right), G=\Gamma_{0}^{+}(n)$. By Theorem 3.1,

$$
128>\left[\Gamma_{0}^{+}(f B \mid B): \Gamma_{0}^{+}(n)\right] \prod_{p \mid f}(p+1) / 2 .
$$

It is clear that the choices of $B$ are finite. Since the signature of $\Gamma_{0}^{+}(m)$ can be determined for any $m$ (see Section 2 ), the set $E$ can be determined by direct calculation.

Corollary 3.5. Let $m=n h^{2}$, where $h$ is the largest divisor of 24 such that $h^{2} \mid m$. Then $m \in \Omega$ if and only if $n \in E$. In particular, $\Omega$ is finite.

Proof: Suppose $m \in \Omega$. Let $m=n h^{2}$, where $h$ is the largest divisor of 24 such that $h^{2} \mid m$. Since $g\left(\Gamma_{0}^{+}(n h \mid h)\right)=$ 
0 , we have $g\left(\Gamma_{0}^{+}(n)\right)=0$. Hence $n \in E$. By Lemma 3.4, the choices of $n$ are finite. Since $h$ is a divisor of 24 and $m=n h^{2}$, the set $\Omega$ is finite.

\subsection{The Set $\Delta$ Is Finite}

$G \in \Delta$ if and only if $\Gamma_{0}(m) \leq G \leq N\left(\Gamma_{0}(m)\right), m \in \Omega$. Since both $\Omega$ and $N\left(\Gamma_{0}(m)\right) / \Gamma_{0}(m)$ are finite, $\Delta$ is finite. Further, the following holds.

Proposition 3.6. Suppose that $G \in \Delta$. Then $\Gamma_{0}(m) \leq$ $G \leq N\left(\Gamma_{0}(m)\right)$ for some $m \in \Omega$. Let $m=n h^{2}$, where $h$ is the largest divisor of $m$ such that $h^{2} \mid m$. Then $N\left(\Gamma_{0}(m)\right)=\Gamma_{0}^{+}(n h \mid h), n \in E$. Further, $64 / 3 \chi\left(\Gamma_{0}^{+}(n h \mid h)\right)>\left[\Gamma_{0}^{+}(n h \mid h): G\right]$. If

$$
\left(\begin{array}{ll}
1 & 1 \\
0 & 1
\end{array}\right)
$$

generates the stabiliser of the infinite cusp of $G$, then

$$
\begin{aligned}
\frac{64}{3 \chi\left(\Gamma_{0}^{+}(n h \mid h)\right)} & >\left[\Gamma_{0}^{+}(n h \mid h): G\right] \\
& \geq\left[\Gamma_{0}^{+}(n h \mid h)_{\infty}: G_{\infty}\right]=h .
\end{aligned}
$$

Proof: Since $g(G)=0$, we have that $\Gamma_{0}^{+}(n h \mid h)$ is of genus 0 . It follows that $\Gamma_{0}^{+}(n)$ is of genus zero. By Lemma

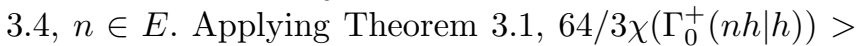
$\left[\Gamma_{0}^{+}(n h \mid h): G\right]$. The rest follows easily.

Proposition 3.6 is useful if one wants to investigate groups $G$ that satisfy

$$
G_{\infty}=\left\langle\left(\begin{array}{ll}
1 & 1 \\
0 & 1
\end{array}\right)\right\rangle
$$

Example 3.7. Suppose that $m=119 \cdot 24^{2} \in \Omega, \Gamma_{0}(m) \leq$ $G \leq N\left(\Gamma_{0}(m)\right), G \in \Delta$. Then $32 / 9=64 / 3 \chi\left(\Gamma_{0}^{+}(119\right.$. $\left.24 \mid 24))>\left[\Gamma_{0}^{+}(119 \cdot 24 \mid 24)\right): G\right]$. As a consequence, $G$ is a maximal subgroup of $\Gamma_{0}^{+}(119 \cdot 24 \mid 24)$. Applying Proposition $3.6, G_{\infty}$ cannot be

$$
\left(\begin{array}{ll}
1 & 1 \\
0 & 1
\end{array}\right)
$$

In summary, $\Gamma_{0}\left(119 \cdot 24^{2}\right) \leq N\left(\Gamma_{0}\left(119 \cdot 24^{2}\right)\right)$ possesses no subgroups $G$ such that $g(G)=0$ and

$$
G_{\infty}=\left(\begin{array}{cc}
1 & 1 \\
0 & 1
\end{array}\right)
$$

\section{THE DETERMINATION OF $\Delta$}

Let $m=n h^{2}$, where $h$ is the largest divisor of 24 such that $h^{2} \mid m$. By Corollary 3.5, $m \in \Omega$ if and only if $n \in E$, where $E$ is the collection of the following 64 numbers: 1, $2,3,5,6,7,10,11,13,14,15,17,19,21,22,23,26,29$, $30,31,33,34,35,38,39,41,42,46,47,51,55,59,62,66$, $69,70,71,78,87,94,95,105,110,119,4,8,9,12,16$, 18, 20, 24, 25, 27, 32, 36, 44, 45, 49, 50, 54, 56, 60, 92. For each $m \in \Omega$, we may now apply Theorem 2.5 to all the intermediate groups of $\Gamma_{0}(m) \leq N\left(\Gamma_{0}(m)\right)$ to determine the set $\left\{G: g(G)=0, \Gamma_{0}(m) \leq G \leq N\left(\Gamma_{0}(m)\right)\right\}$. This is achieved by a computer program written in GAP 4.3. This program, which is available upon request, computes the genera of maximal subgroups recursively until all subgroups of genus 0 are obtained. A branch is cut off when a subgroup is of positive genus or the index of the subgroup exceeds the bound (see Remark 4.1). The last step of the program checks the conjugation in $N\left(\Gamma_{0}(m)\right)$ and the output is the list of all proper subgroups of genus 0 . A few useful remarks can be found in the following:

(i) a complete set of representatives of cusps of $\Gamma_{0}\left(n h^{2}\right)$ can be found in Section 6 .

(ii) denote the set in (i) by $T$. Let

$$
\begin{aligned}
& x=\left(\begin{array}{cc}
1 & 1 / h \\
0 & 1
\end{array}\right), \\
& y=\left(\begin{array}{cc}
1 & 0 \\
n h & 1
\end{array}\right) .
\end{aligned}
$$

By the results of Akbas and Singerman [Akbas and Singerman 90], $\Gamma_{0}^{+}(n h \mid h) / \Gamma_{0}\left(n h^{2}\right)$ is generated by $x$, $y$ and $w_{e}, e \| n$. By Lemma $2.3, \Gamma_{0}^{+}(n h \mid h) / \Gamma_{0}\left(n h^{2}\right) \cong$ $\rho\left(\Gamma_{0}^{+}(n h \mid h)\right)$, where $\rho\left(\Gamma_{0}^{+}(n h \mid h)\right)$ is a subgroup of the symmetric group $S_{T}$.

(iii) a complete list of nonconjugate elliptic subgroups of order $2,3,4,6$ of $\Gamma_{0}^{+}(n h \mid h)$, where $n \in E$, can be found in Section 5. Their permutation representations can be determined by our results in Section 6 .

Remark 4.1. Take $m=119 \cdot 24^{2}$, for example, $\Gamma_{0}^{+}(119$. $24 \mid 24)$ is of order 4608. Proposition 3.6 implies that if $G$ is a subgroup of $N\left(\Gamma_{0}\left(119 \cdot 24^{2}\right)\right)$ of genus 0 , then the index $\left[N\left(\Gamma_{0}\left(119 \cdot 24^{2}\right)\right): G\right]$ is at most $32 / 9$.

\subsection{The Output}

For each $m=n h^{2} \in \Omega$, a complete list of intermediate groups of $\Gamma_{0}(m) \leq N\left(\Gamma_{0}(m)\right)$ of genus 0 can be found on the following web site: www.math.nus.edu.sg/ matlml/. 


\begin{tabular}{|l|l|l|l|l|}
\hline Group & Index & Width of $\infty$ & {$\left[c, v_{2}, v_{3}, v_{4}, v_{6}\right]$} & Generators \\
\hline 1.4 .8 .1 & 6 & 1 & {$[2,2,0,0,0]$} & {$[3,-1 / 2,-4,1],[1,0,-8,1]$} \\
\hline 1.4 .8 .2 & 6 & 1 & {$[2,2,0,0,0]$} & {$[2,-1 / 4,-12,2],[5,-1 / 2,-8,1]$} \\
\hline 1.4 .8 .3 & 6 & $1 / 2$ & {$[2,2,0,0,0]$} & {$[-1,1 / 4,-8,1],[1,-1 / 2,0,1]$} \\
\hline
\end{tabular}

TABLE 1.

\begin{tabular}{|l|l|l|l|l|}
\hline Group & Index & Width of $\infty$ & {$\left[c, v_{2}, v_{3}, v_{4}, v_{6}\right]$} & Generator \\
\hline 1.4 .9 .1 & 12 & 1 & {$[3,2,0,0,0]$} & {$[3,-1 / 2,-4,1]$} \\
\hline 1.4 .9 .2 & 12 & 1 & {$[3,2,0,0,0]$} & {$[-1,-1 / 2,4,1]$} \\
\hline 1.4 .9 .3 & 12 & 1 & {$[3,2,0,0,0]$} & {$[-1,1 / 4,-8,1]$} \\
\hline 1.4 .9 .4 & 12 & 1 & {$[3,2,0,0,0]$} & {$[3,-1 / 4,-8,1]$} \\
\hline 1.4 .9 .5 & 12 & 1 & {$[3,2,0,0,0]$} & {$[2,-1 / 4,-12,2]$} \\
\hline 1.4 .9 .6 & 12 & 1 & {$[3,2,0,0,0]$} & {$[0,1 / 4,-4,0]$} \\
\hline
\end{tabular}

TABLE 2.

All the intermediate groups of width 1 (at $\infty$ ) are also listed on this web site. Notations used in our lists can be found in the following:

(i) intermediate groups of genus 0 of $\Gamma_{0}\left(n h^{2}\right) \leq$ $N\left(\Gamma_{0}\left(n h^{2}\right)\right)=\Gamma_{0}^{+}(n h \mid h)$ form $t$ conjugacy classes. They are listed as n.h.1, n.h.2, $\cdots$, n.h.t.

(ii) suppose that the $r$ th class has $k$ groups. Then these groups are listed as n.h.r.1, n.h.r.2, $\cdots$, n.h.r.k. The groups are listed according to their width at $\infty$.

(iii) class with $*\left(n . h . r^{*}\right)$ in $\Gamma_{0}^{+}(n h \mid h)$ possesses a single member only. As a consequence, this group is normal in $\Gamma_{0}^{+}(n h \mid h)$. Take 1.2.1*, for example, the * means that the class 1.2.1 possesses a single member 1.2.1.1 only and the group 1.2.1.1 is a normal subgroup of $\Gamma_{0}^{+}(2 \mid 2)$.

See the following for two easy examples.

Example 4.2. Table 1 gives all the intermediate groups of genus 0 of the 8 th conjugacy class of $\Gamma_{0}(16) \leq$ $N\left(\Gamma_{0}(16)\right)=\Gamma_{0}^{+}(4 \mid 4)$.

\section{Remark 4.3.}

(i) The $c$ in the forth column are the number of cusps of the group 1.4.8.x.

(ii) The last column is a set of generators of 1.4.8.x modulo $\Gamma_{0}(16)$. The tuple $[a, b, c, d]$ represents the matrix

$$
\frac{1}{\sqrt{a d-b c}}\left(\begin{array}{cc}
a & b \\
c & d
\end{array}\right)
$$

(iii) The last column gives a set of generators modulo $\Gamma_{0}(16)$.

(iv) Width is not invariant under conjugation.

Example 4.4. Table 2 gives all the groups of genus 0 , width 1 (at $\infty$ ) of the 9th conjugacy class of the intermediate groups of $\Gamma_{0}(16) \leq N\left(\Gamma_{0}(16)\right)=\Gamma_{0}^{+}(4 \mid 4)$.

Remark 4.5. The above actually gives all the groups in the 9 th class.

\subsection{The Table}

We shall now give the number of intermediate groups of genus zero of $\Gamma_{0}\left(n h^{2}\right) \leq N\left(\Gamma_{0}\left(n h^{2}\right)\right)$ in Table 3 . The entry $52(177,25)$ for $n=2, h=12\left(2.12^{2}=288\right)$ means that

(i) $m=n h^{2}=288$, there are 52 conjugacy classes of intermediate groups of $\Gamma_{0}(288)<N\left(\Gamma_{0}(288)\right)$ of genus zero,

(ii) there are altogether 177 intermediate groups of $\Gamma_{0}(288)<N\left(\Gamma_{0}(288)\right)$ of genus zero; 25 of them have width 1 at infinity.

The remaining entries can be read similarly. An entry ** denotes that $h$ is not the largest divisor of 24 such that $h^{2}$ divides $n h^{2}$. Note that there are $419 m$ and $64 n$. 


\begin{tabular}{|c|c|c|c|c|c|c|c|c|}
\hline$(n, h)$ & 1 & 2 & 3 & 4 & 6 & 8 & 12 & 24 \\
\hline 1 & $1(1,1)$ & $4(6,4)$ & $5(10,8)$ & $11(30,21)$ & $20(84,56)$ & $17(70,32)$ & $34(166,42)$ & $41(209,2)$ \\
\hline 2 & $2(2,2)$ & $8(10,7)$ & $11(30,26)$ & $20(42,27)$ & $34(119,67)$ & $25(60,16)$ & $52(177,25)$ & $57(195,0)$ \\
\hline 3 & $2(2,2)$ & $10(16,12)$ & $7(12,9)$ & $21(53,31)$ & $24(55,22)$ & $24(68,10)$ & $37(99,6)$ & $40(114,0)$ \\
\hline 4 & $* *$ & $* *$ & $* *$ & $* *$ & $* *$ & $18(30,10)$ & $* *$ & $32(65,1)$ \\
\hline 5 & $2(2,2)$ & $6(12,8)$ & $6(13,10)$ & $11(29,14)$ & $12(30,6)$ & $12(32,2)$ & $17(47,0)$ & $18(50,0)$ \\
\hline 6 & $5(5,5)$ & $19(27,19)$ & $12(30,21)$ & $32(59,27)$ & $32(66,14)$ & $35(69,10)$ & $48(111,13)$ & $51(121,0)$ \\
\hline 7 & $2(2,2)$ & $7(9,6)$ & $4(6,4)$ & $8(12,2)$ & $11(18,4)$ & $8(12,0)$ & $12(21,0)$ & $12(21,0)$ \\
\hline 8 & $* *$ & $* *$ & $* *$ & $* *$ & $* *$ & $7(9,1)$ & $* *$ & $8(13,0)$ \\
\hline 9 & $* *$ & $* *$ & $5(7,5)$ & $* *$ & $10(18,1)$ & $* *$ & $11(21,0)$ & $11(21,0)$ \\
\hline 10 & $5(5,5)$ & $14(16,10)$ & $8(12,7)$ & $19(24,8)$ & $20(30,7)$ & $19(24,0)$ & $25(38,0)$ & $25(38,0)$ \\
\hline 11 & $1(1,1)$ & $3(5,3)$ & $2(5,3)$ & $5(12,6)$ & $4(9,0)$ & $5(12,0)$ & $6(16,0)$ & $6(16,0)$ \\
\hline 12 & $* *$ & $* *$ & $* *$ & $* *$ & $* *$ & $17(21,0)$ & $* *$ & $20(25,0)$ \\
\hline 13 & $2(2,2)$ & $3(3,1)$ & $3(3,1)$ & $3(3,0)$ & $4(4,0)$ & $3(3,0)$ & $4(4,0)$ & $4(4,0)$ \\
\hline 14 & $3(3,3)$ & $8(10,6)$ & $6(10,7)$ & $11(15,5)$ & $11(17,0)$ & $11(15,0)$ & $14(22,0)$ & $14(22,0)$ \\
\hline 15 & $3(3,3)$ & $9(15,10)$ & $5(7,4)$ & $10(18,2)$ & $11(19,0)$ & $10(18,0)$ & $12(22,0)$ & $12(22,0)$ \\
\hline 16 & $* *$ & $* *$ & $* *$ & $* *$ & $* *$ & $3(3,0)$ & $* *$ & $3(3,0)$ \\
\hline 17 & $1(1,1)$ & $2(2,1)$ & $1(1,0)$ & $2(2,0)$ & $2(2,0)$ & $2(2,0)$ & $2(2,0)$ & $2(2,0)$ \\
\hline 18 & $* *$ & $* *$ & $7(9,4)$ & $* *$ & $10(14,0)$ & ** & $10(14,0)$ & $10(14,0)$ \\
\hline 19 & $1(1,1)$ & $2(2,1)$ & $2(2,1)$ & $2(2,0)$ & $3(3,0)$ & $2(2,0)$ & $3(3,0)$ & $3(3,0)$ \\
\hline 20 & $* *$ & $* *$ & $* *$ & $* *$ & $* *$ & $7(7,0)$ & $* *$ & $7(7,0)$ \\
\hline 21 & $3(3,3)$ & $6(6,3)$ & $4(4,1)$ & $6(6,0)$ & $8(8,1)$ & $6(6,0)$ & $8(8,0)$ & $8(8,0)$ \\
\hline 22 & $2(2,2)$ & $5(5,3)$ & $2(2,0)$ & $5(5,0)$ & $5(5,0)$ & $5(5,0)$ & $5(5,0)$ & $5(5,0)$ \\
\hline 23 & $1(1,1)$ & $2(4,2)$ & $1(1,0)$ & $2(4,0)$ & $2(4,0)$ & $2(4,0)$ & $2(4,0)$ & $2(4,0)$ \\
\hline 24 & $* *$ & $* *$ & $* *$ & $* *$ & $* *$ & $4(4,0)$ & $* *$ & $4(4,0)$ \\
\hline 25 & $2(2,2)$ & $2(2,0)$ & $2(2,0)$ & $2(2,0)$ & $2(2,0)$ & $2(2,0)$ & $2(2,0)$ & $2(2,0)$ \\
\hline 26 & $2(2,2)$ & $5(5,3)$ & $2(5,0)$ & $6(6,1)$ & $5(5,0)$ & $6(6,0)$ & $6(6,0)$ & $6(6,0)$ \\
\hline 27 & $* *$ & $* *$ & $1(1,0)$ & $* *$ & $1(1,0)$ & $* *$ & $1(1,0)$ & $1(1,0)$ \\
\hline 29 & $1(1,1)$ & $2(2,1)$ & $1(1,0)$ & $2(2,0)$ & $2(2,0)$ & $2(2,0)$ & $2(2,0)$ & $2(2,0)$ \\
\hline 30 & $6(6,6)$ & $11(13,6)$ & $8(10,4)$ & $11(13,0)$ & $13(17,0)$ & $11(13,0)$ & $13(17,0)$ & $13(17,0)$ \\
\hline 31 & $1(1,1)$ & $1(1,0)$ & $2(2,1)$ & $1(1,0)$ & $2(2,0)$ & $1(1,0)$ & $2(2,0)$ & $2(2,0)$ \\
\hline 32 & $* *$ & $* *$ & $* *$ & $* *$ & $* *$ & $1(1,0)$ & $* *$ & $1(1,0)$ \\
\hline 33 & $2(2,2)$ & $3(3,1)$ & $2(2,0)$ & $3(3,0)$ & $3(3,0)$ & $3(3,0)$ & $3(3,0)$ & $3(3,0)$ \\
\hline 34 & $1(1,1)$ & $2(2,1)$ & $1(1,0)$ & $2(2,0)$ & $2(2,0)$ & $2(2,0)$ & $2(2,0)$ & $2(2,0)$ \\
\hline 35 & $2(2,2)$ & $3(3,1)$ & $2(2,0)$ & $3(3,0)$ & $3(3,0)$ & $3(3,0)$ & $3(3,0)$ & $3(3,0)$ \\
\hline 36 & $* *$ & $* *$ & $* *$ & $* *$ & $* *$ & $* *$ & $* *$ & $4(4,0)$ \\
\hline 38 & $1(1,1)$ & $2(2,1)$ & $1(1,0)$ & $2(2,0)$ & $2(2,0)$ & $2(2,0)$ & $2(2,0)$ & $2(2,0)$ \\
\hline 39 & $2(2,2)$ & $2(2,0)$ & $3(3,1)$ & $2(2,0)$ & $3(3,0)$ & $2(2,0)$ & $3(3,0)$ & $3(3,0)$ \\
\hline 41 & $1(1,1)$ & $2(2,1)$ & $1(1,0)$ & $2(2,0)$ & $2(2,0)$ & $2(2,0)$ & $2(2,0)$ & $2(2,0)$ \\
\hline 42 & $3(3,3)$ & $6(6,3)$ & $3(3,0)$ & $6(6,0)$ & $6(6,0)$ & $6(6,0)$ & $6(6,0)$ & $6(6,0)$ \\
\hline 44 & $* *$ & $* *$ & $* *$ & $* *$ & $* *$ & $2(2,0)$ & $* *$ & $2(2,0)$ \\
\hline 45 & $* *$ & $* *$ & $1(1,0)$ & $* *$ & $1(1,0)$ & $* *$ & $1(1,0)$ & $1(1,0)$ \\
\hline 46 & $2(2,2)$ & $2(2,0)$ & $2(2,0)$ & $2(2,0)$ & $2(2,0)$ & $2(2,0)$ & $2(2,0)$ & $2(2,0)$ \\
\hline 47 & $1(1,1)$ & $1(1,0)$ & $1(1,0)$ & $1(1,0)$ & $1(1,0)$ & $1(1,0)$ & $1(1,0)$ & $1(1,0)$ \\
\hline 49 & $1(1,1)$ & $1(1,0)$ & $1(1,0)$ & $1(1,0)$ & $1(1,0)$ & $1(1,0)$ & $1(1,0)$ & $1(1,0)$ \\
\hline 50 & $2(2,2)$ & $2(2,0)$ & $2(2,0)$ & $2(2,0)$ & $2(2,0)$ & $2(2,0)$ & $2(2,0)$ & $2(2,0)$ \\
\hline 51 & $1(1,1)$ & $2(2,1)$ & $1(1,0)$ & $2(2,0)$ & $2(2,0)$ & $2(2,0)$ & $2(2,0)$ & $2(2,0)$ \\
\hline 54 & $* *$ & $* *$ & $1(1,0)$ & $* *$ & $1(1,0)$ & $* *$ & $1(1,0)$ & $1(1,0)$ \\
\hline 55 & $1(1,1)$ & $1(1,0)$ & $1(1,0)$ & $1(1,0)$ & $1(1,0)$ & $1(1,0)$ & $1(1,0)$ & $1(1,0)$ \\
\hline 56 & $* *$ & $* *$ & $* *$ & $* *$ & $* *$ & $1(1,0)$ & $* *$ & $1(1,0)$ \\
\hline 59 & $1(1,1)$ & $1(1,0)$ & $1(1,0)$ & $1(1,0)$ & $1(1,0)$ & $1(1,0)$ & $1(1,0)$ & $1(1,0)$ \\
\hline 60 & $* *$ & $* *$ & $* *$ & $* *$ & $* *$ & $4(4,0)$ & $* *$ & $4(4,0)$ \\
\hline 62 & $1(1,1)$ & $1(1,0)$ & $1(1,0)$ & $1(1,0)$ & $1(1,0)$ & $1(1,0)$ & $1(1,0)$ & $1(1,0)$ \\
\hline 66 & $2(2,2)$ & $3(3,1)$ & $2(2,0)$ & $3(3,0)$ & $3(3,0)$ & $3(3,0)$ & $3(3,0)$ & $3(3,0)$ \\
\hline 69 & $1(1,1)$ & $1(1,0)$ & $1(1,0)$ & $1(1,0)$ & $1(1,0)$ & $1(1,0)$ & $1(1,0)$ & $1(1,0)$ \\
\hline 70 & $2(2,2)$ & $3(3,1)$ & $2(2,0)$ & $3(3,0)$ & $3(3,0)$ & $3(3,0)$ & $3(3,0)$ & $3(3,0)$ \\
\hline 71 & $1(1,1)$ & $1(1,0)$ & $1(1,0)$ & $1(1,0)$ & $1(1,0)$ & $1(1,0)$ & $1(1,0)$ & $1(1,0)$ \\
\hline 78 & $2(2,2)$ & $2(2,0)$ & $2(2,0)$ & $2(2,0)$ & $2(2,0)$ & $2(2,0)$ & $2(2,0)$ & $2(2,0)$ \\
\hline 87 & $1(1,1)$ & $1(1,0)$ & $1(1,0)$ & $1(1,0)$ & $1(1,0)$ & $1(1,0)$ & $1(1,0)$ & $1(1,0)$ \\
\hline 92 & $* *$ & $* *$ & $* *$ & $* *$ & $* *$ & $1(1,0)$ & $* *$ & $1(1,0)$ \\
\hline 94 & $1(1,1)$ & $1(1,0)$ & $1(1,0)$ & $1(1,0)$ & $1(1,0)$ & $1(1,0)$ & $1(1,0)$ & $1(1,0)$ \\
\hline 95 & $1(1,1)$ & $1(1,0)$ & $1(1,0)$ & $1(1,0)$ & $1(1,0)$ & $1(1,0)$ & $1(1,0)$ & $1(1,0)$ \\
\hline 105 & $1(1,1)$ & $1(1,0)$ & $1(1,0)$ & $1(1,0)$ & $1(1,0)$ & $1(1,0)$ & $1(1,0)$ & $1(1,0)$ \\
\hline 110 & $1(1,1)$ & $1(1,0)$ & $1(1,0)$ & $1(1,0)$ & $1(1,0)$ & $1(1,0)$ & $1(1,0)$ & $1(1,0)$ \\
\hline 119 & $1(1,1)$ & $1(1,0)$ & $1(1,0)$ & $1(1,0)$ & $1(1,0)$ & $1(1,0)$ & $1(1,0)$ & $1(1,0)$ \\
\hline
\end{tabular}

TABLE 3. 


\begin{tabular}{|c|c|c|c|c|c|c|c|c|c|c|c|c|c|}
\hline$n$ & $v_{2}$ & $v_{3}$ & $v_{4}$ & $v_{6}$ & $c$ & $g$ & $n$ & $v_{2}$ & $v_{3}$ & $v_{4}$ & $v_{6}$ & $c$ & $g$ \\
\hline 1 & 1 & 1 & 0 & 0 & 1 & 0 & 34 & 5 & 0 & 1 & 0 & 1 & 0 \\
\hline 2 & 1 & 0 & 1 & 0 & 1 & 0 & 35 & 6 & 0 & 0 & 0 & 1 & 0 \\
\hline 3 & 1 & 0 & 0 & 1 & 1 & 0 & 36 & 2 & 0 & 0 & 0 & 4 & 0 \\
\hline 4 & 1 & 0 & 0 & 0 & 2 & 0 & 38 & 7 & 0 & 0 & 0 & 1 & 0 \\
\hline 5 & 3 & 0 & 0 & 0 & 1 & 0 & 39 & 5 & 0 & 0 & 1 & 1 & 0 \\
\hline 6 & 3 & 0 & 0 & 0 & 1 & 0 & 41 & 9 & 0 & 0 & 0 & 1 & 0 \\
\hline 7 & 2 & 1 & 0 & 0 & 1 & 0 & 42 & 6 & 0 & 0 & 0 & 1 & 0 \\
\hline 8 & 2 & 0 & 0 & 0 & 2 & 0 & 44 & 6 & 0 & 0 & 0 & 2 & 0 \\
\hline 9 & 2 & 0 & 0 & 0 & 2 & 0 & 45 & 6 & 0 & 0 & 0 & 2 & 0 \\
\hline 10 & 2 & 0 & 1 & 0 & 1 & 0 & 46 & 8 & 0 & 0 & 0 & 1 & 0 \\
\hline 11 & 4 & 0 & 0 & 0 & 1 & 0 & 47 & 10 & 0 & 0 & 0 & 1 & 0 \\
\hline 12 & 2 & 0 & 0 & 0 & 2 & 0 & 49 & 4 & 1 & 0 & 0 & 4 & 0 \\
\hline 13 & 3 & 1 & 0 & 0 & 1 & 0 & 50 & 4 & 0 & 1 & 0 & 3 & 0 \\
\hline 14 & 4 & 0 & 0 & 0 & 1 & 0 & 51 & 8 & 0 & 0 & 0 & 1 & 0 \\
\hline 15 & 4 & 0 & 0 & 0 & 1 & 0 & 54 & 7 & 0 & 0 & 0 & 3 & 0 \\
\hline 16 & 2 & 0 & 0 & 0 & 3 & 0 & 55 & 8 & 0 & 0 & 0 & 1 & 0 \\
\hline 17 & 5 & 0 & 0 & 0 & 1 & 0 & 56 & 8 & 0 & 0 & 0 & 2 & 0 \\
\hline 18 & 3 & 0 & 0 & 0 & 2 & 0 & 59 & 12 & 0 & 0 & 0 & 1 & 0 \\
\hline 19 & 4 & 1 & 0 & 0 & 1 & 0 & 60 & 6 & 0 & 0 & 0 & 2 & 0 \\
\hline 20 & 3 & 0 & 0 & 0 & 2 & 0 & 62 & 10 & 0 & 0 & 0 & 1 & 0 \\
\hline 21 & 3 & 0 & 0 & 1 & 1 & 0 & 66 & 8 & 0 & 0 & 0 & 1 & 0 \\
\hline 22 & 5 & 0 & 0 & 0 & 1 & 0 & 69 & 10 & 0 & 0 & 0 & 1 & 0 \\
\hline 23 & 6 & 0 & 0 & 0 & 1 & 0 & 70 & 8 & 0 & 0 & 0 & 1 & 0 \\
\hline 24 & 4 & 0 & 0 & 0 & 2 & 0 & 71 & 14 & 0 & 0 & 0 & 1 & 0 \\
\hline 25 & 3 & 0 & 0 & 0 & 3 & 0 & 78 & 9 & 0 & 0 & 0 & 1 & 0 \\
\hline 26 & 4 & 0 & 1 & 0 & 1 & 0 & 87 & 12 & 0 & 0 & 0 & 1 & 0 \\
\hline 27 & 4 & 0 & 0 & 0 & 3 & 0 & 92 & 12 & 0 & 0 & 0 & 2 & 0 \\
\hline 29 & 7 & 0 & 0 & 0 & 1 & 0 & 94 & 14 & 0 & 0 & 0 & 1 & 0 \\
\hline 30 & 5 & 0 & 0 & 0 & 1 & 0 & 95 & 12 & 0 & 0 & 0 & 1 & 0 \\
\hline 31 & 6 & 1 & 0 & 0 & 1 & 0 & 105 & 10 & 0 & 0 & 0 & 1 & 0 \\
\hline 32 & 4 & 0 & 0 & 0 & 4 & 0 & 110 & 11 & 0 & 0 & 0 & 1 & 0 \\
\hline 33 & 6 & 0 & 0 & 0 & 1 & 0 & 119 & 14 & 0 & 0 & 0 & 1 & 0 \\
\hline
\end{tabular}

TABLE 4. Signature of $\Gamma_{0}^{+}(n), n \in E$.

Recall that $E$ is the set of all $n$ such that $g\left(\Gamma_{0}^{+}(n)\right)=0$.

(i) The signature of the groups in Table 4 determined by Riemann-Hurwitz formula and results of [Akbas and Singerman 92], [Conway 79], and [Maclachlan 81].

(ii) $\Gamma_{0}^{+}(25), \Gamma_{0}^{+}(49)$, and $\Gamma_{0}^{+}(50)$ are known as the ghost classes of the Monster. (see [Conway 79] for more detail).
For Table 5,

(i) $n+e+f+\cdots$ is the group $\Gamma_{0}(n)+w_{e}+w_{f}+\cdots=$ $\left\langle\Gamma_{0}(n), w_{e}, w_{f}, \cdots\right\rangle$ and $n+$ is the group generated by $\Gamma_{0}(n)$ and all the Atkin-Lehner involutions of $\Gamma_{0}(n)$. Table 5 has 123 groups.

(ii) The completeness of Table 5 was first proved by P. G. Kluit in his thesis [Kluit 79]. 


\begin{tabular}{|c|c|}
\hline 1 & $34+$ \\
\hline $2,2+$ & $35+35,35+$ \\
\hline $3,3+$ & $36+4,36+36,36+$ \\
\hline $4,4+$ & $38+$ \\
\hline $5,5+$ & $39+39,39+$ \\
\hline $6,6+2,6+3,6+6,6+$ & $41+$ \\
\hline $7,7+$ & $42+3+14,42+6+14,42+$ \\
\hline $8,8+$ & $44+$ \\
\hline $9,9+$ & $45+$ \\
\hline $10,10+2,10+5,10+10,10+$ & $46+23,46+$ \\
\hline $11+$ & $47+$ \\
\hline $12,12+3,12+4,12+12,12+$ & $49+$ \\
\hline $13,13+$ & $50+50,50+$ \\
\hline $14+7,14+14,14+$ & $51+$ \\
\hline $15+5,15+15,15+$ & $54+$ \\
\hline $16,16+$ & $55+$ \\
\hline $17+$ & $56+$ \\
\hline $18,18+2,18+9,18+18,18+$ & $59+$ \\
\hline $19+19$ & $60+4+15,60+15+20,60+$ \\
\hline $20+4,20+20,20+$ & $62+$ \\
\hline $21+3,21+21,21+$ & $66+6+11,66+$ \\
\hline $22+11,22+$ & $69+$ \\
\hline $23+$ & $70+10+14,70+$ \\
\hline $24+8,24+24,24+$ & $71+$ \\
\hline $25,25+$ & $78+26+39,78+$ \\
\hline $26+26,26+$ & $87+$ \\
\hline $27+$ & $92+$ \\
\hline $28+7,28+$ & $94+$ \\
\hline $29+$ & $95+$ \\
\hline $30+15,30+5,6,30+3+5,30+2+15,30+6+10,30+$ & $105+$ \\
\hline $31+$ & $110+$ \\
\hline $32+$ & $119+$ \\
\hline $33+11,33+$ & \\
\hline
\end{tabular}

TABLE 5. $G$ such that $g(G)=0, \Gamma_{0}(n) \leq G \leq \Gamma_{0}^{+}(n)$.

\section{ELLIPTIC PERIODS}

Let $\sigma \in \Gamma_{0}^{+}(n)$ be an element of order $k$. $\langle\sigma\rangle$ is an elliptic subgroup of order $k$ of $\Gamma_{0}^{+}(n)$ if and only if $\langle\sigma\rangle$ is a maximal cyclic subgroup of $\Gamma_{0}^{+}(n)$. Since the entries of members of $\Gamma_{0}^{+}(n)$ are elements of $\mathbb{Q}(\sqrt{a}, a \in \mathbb{N})$, one can show easily that $k$ is either $2,3,4$, or 6 . By results of Maclachlan and Akbas-Singerman ([Maclachlan 81], [Akbas and Singerman 92]), the number of nonconjugate elliptic subgroups of order $k(k=3,4$, or 6$)$ is either 0 or 1 .
Further,

(i) $\Gamma_{0}^{+}(n)$ has a unique conjugacy class of elliptic subgroups of order 3 if and only if all the prime divisors of $n$ are of the form $3 k+1$,

(ii) $\Gamma_{0}^{+}(n)$ has a unique conjugacy class of elliptic subgroups of order 4 if and only if $n$ is even and all the prime divisors of $n / 2$ are of the form $4 k+1$,

(iii) $\Gamma_{0}^{+}(n)$ has a unique conjugacy class of elliptic subgroups of order 6 if and only if $n$ is a multiple of 
3 and all the prime divisors of $n / 3$ are of the form $3 k+1$.

Since an element is of order $3(4,6)$ if and only if the trace is $\pm 1( \pm \sqrt{2}, \pm \sqrt{3})$, a representative of an elliptic element of order $3(4,6)$ of $\Gamma_{0}^{+}(n)$ can be obtained easily (see (i), (ii) and (iii) of Remark 5.4). It is clear that an element $g$ is of order 2 if and only if the trace of $g$ is zero. Let

$$
\sigma=\left(\begin{array}{cc}
a \sqrt{e} & -b / \sqrt{e} \\
c n / \sqrt{e} & -a \sqrt{e}
\end{array}\right), \tau=\left(\begin{array}{cc}
x \sqrt{f} & y / \sqrt{f} \\
z n / \sqrt{f} & w \sqrt{f}
\end{array}\right)
$$

$\in \Gamma_{0}^{+}(n)$. Then the $(2,1)$ and $(1,2)$ entries of $\tau \sigma \tau^{-1}$ are given by the following:

$$
\begin{aligned}
& \left(c f w^{2}+2 a z w e+b n z^{2} / f\right) n / \sqrt{e}, \\
& -\left(b f x^{2}+2 a x y e+c n y^{2} / f\right) / \sqrt{e} .
\end{aligned}
$$

Direct calculation shows that

$$
\begin{aligned}
c f w^{2}+2 a z w e+b n z^{2} / f & =c f(w+a e z / c f)^{2}+e z^{2} / c f, \\
b f x^{2}+2 a x y e+c n y^{2} / f & =b f(x+a e y / b f)^{2}+e y^{2} / b f .
\end{aligned}
$$

Since $f, e>0$ (replace $b, c$ by $-b,-c$ if necessary), we may assume that the quadratic forms

$$
c f w^{2}+2 a z w e+b n z^{2} / f, b f x^{2}+2 a x y e+c n y^{2} / f
$$

are positive definite. As a consequence, whether $\sigma_{1}, \sigma_{2} \in$ $w_{e} \Gamma_{0}(n)\left(o\left(\sigma_{1}\right)=o\left(\sigma_{2}\right)=2\right)$ are conjugate to each other in $\Gamma_{0}^{+}(n)$ can be determined by direct calculation. Applying the results of Maclachlan [Maclachlan 81], the number of nonconjugate elliptic subgroups of order 2 in $w_{e} \Gamma_{0}(n)$ (where $n$ is square-free) is known. Hence representatives of nonconjugate elliptic subgroups of order 2 in $w_{e} \Gamma_{0}(n)$ can be determined. In summary, a complete set of representatives of nonconjugate elliptic subgroups of order 2 of $\Gamma_{0}^{+}(n)$, where $n$ is square-free, can be obtained.

Remark 5.1. In the case $n$ is not square-free, let $n=f B^{2}$, where $f$ is square-free, then $f \in F$ (see Section 3.1) and $\Gamma_{0}^{+}(n) \leq \Gamma_{0}^{+}(f B \mid B)$. The set of elliptic subgroups of order 2 of $\Gamma_{0}^{+}(f B \mid B)$ can be determined by the above and the number of nonconjugating elliptic subgroups of order 2 of $\Gamma_{0}^{+}(n)$ can be determined by applying (ii) $-(\mathrm{v})$ of Section 2.2.

Example 5.2. An element in $\Gamma_{0}^{+}(11)$ is of the form $\sigma$ or $\sigma w_{11}$, where $\sigma \in \Gamma_{0}(11)$,

$$
w_{11}=\left(\begin{array}{cc}
\sqrt{11} & -4 / \sqrt{11} \\
3 \sqrt{11} & -\sqrt{11}
\end{array}\right) .
$$

Direct calculation shows that

$$
\tau\left(\begin{array}{cc}
\sqrt{11} & -4 / \sqrt{11} \\
3 \sqrt{11} & -\sqrt{11}
\end{array}\right) \tau^{-1}=\left(\begin{array}{cc}
* & -3 / \sqrt{11} \\
4 \sqrt{11} & *
\end{array}\right)
$$

if and only if

$$
\tau= \pm\left(\begin{array}{cc}
3 & -1 \\
-11 & 4
\end{array}\right)
$$

or

$$
\tau= \pm\left(\begin{array}{cc}
3 & -1 \\
-11 & 4
\end{array}\right)\left(\begin{array}{cc}
\sqrt{11} & -4 / \sqrt{11} \\
3 \sqrt{11} & -\sqrt{11}
\end{array}\right)
$$

Further,

$$
\begin{aligned}
& \left(\begin{array}{cc}
3 & -1 \\
-11 & 4
\end{array}\right)\left(\begin{array}{cc}
\sqrt{11} & -4 / \sqrt{11} \\
3 \sqrt{11} & -\sqrt{11}
\end{array}\right) \\
& \quad \times\left(\begin{array}{cc}
3 & -1 \\
-11 & 4
\end{array}\right)^{-1}=\left(\begin{array}{cc}
-\sqrt{11} & -3 / \sqrt{11} \\
4 \sqrt{11} & \sqrt{11}
\end{array}\right) .
\end{aligned}
$$

This implies that

$$
\left(\begin{array}{cc}
\sqrt{11} & -4 / \sqrt{11} \\
3 \sqrt{11} & -\sqrt{11}
\end{array}\right) \text { and }\left(\begin{array}{cc}
\sqrt{11} & -3 / \sqrt{11} \\
4 \sqrt{11} & -\sqrt{11}
\end{array}\right)
$$

are not conjugate to each other in $\Gamma_{0}^{+}(11)$.

Example 5.3. The following is a complete set of representatives of nonconjugate elliptic subgroups of order 2 of $\Gamma_{0}^{+}(38)$ :

$$
\begin{aligned}
& \left(\begin{array}{cc}
3 \sqrt{2} & -1 / \sqrt{2} \\
19 \sqrt{2} & -3 \sqrt{2}
\end{array}\right),\left(\begin{array}{cc}
\sqrt{19} & -10 / \sqrt{19} \\
2 \sqrt{19} & -\sqrt{19}
\end{array}\right), \\
& \left(\begin{array}{cc}
\sqrt{19} & -5 / \sqrt{19} \\
4 \sqrt{19} & -\sqrt{19}
\end{array}\right),\left(\begin{array}{cc}
\sqrt{19} & -2 / \sqrt{19} \\
10 \sqrt{19} & -\sqrt{19}
\end{array}\right), \\
& \left(\begin{array}{cc}
0 & -1 / \sqrt{38} \\
\sqrt{38} & 0
\end{array}\right),\left(\begin{array}{cc}
\sqrt{38} & -13 / \sqrt{38} \\
3 \sqrt{38} & -\sqrt{38}
\end{array}\right), \\
& \left(\begin{array}{cc}
\sqrt{38} & -3 / \sqrt{38} \\
13 \sqrt{38} & -\sqrt{38}
\end{array}\right) .
\end{aligned}
$$

Proof: It is well known that $\Gamma_{0}(38)$ has no elements of order 2. Further, by results of Maclachlan [Maclachlan 81], $w_{2} \Gamma_{0}(38), w_{19} \Gamma_{0}(38)$, and $w_{38} \Gamma_{0}(38)$ admit 1,3 , and 3 nonconjugate elliptic subgroups of order 2 , respectively. Direct calculation shows that the above is a complete set of representatives of nonconjugate elliptic subgroups of order 2. Note that our calculation is finite since the forms in (5-1) are positive definite. 


\section{Remark 5.4.}

(i) Suppose that $n \in E$. Then $v_{6}=1$ if and only if $n=$ $3,21,39$. It follows that an involution $\sigma \in w_{3} \Gamma_{0}(n)$ is an elliptic element of $\Gamma_{0}^{+}(n)$ order 2 if and only if it is not conjugate to the cubic of $u \in w_{3} \Gamma_{0}(n)$, where $u$ is elliptic of order 6 as follows:

$$
\begin{aligned}
& \left(\begin{array}{cc}
0 & -1 / \sqrt{3} \\
\sqrt{3} & -\sqrt{3}
\end{array}\right) \in w_{3} \Gamma_{0}(3), \\
& \left(\begin{array}{cc}
\sqrt{3} & -1 / \sqrt{3} \\
7 \sqrt{3} & -2 \sqrt{3}
\end{array}\right) \in w_{3} \Gamma_{0}(21), \\
& \left(\begin{array}{cc}
5 \sqrt{3} & -1 / \sqrt{3} \\
7.13 \sqrt{3} & -6 \sqrt{3}
\end{array}\right) \in w_{3} \Gamma_{0}(39) .
\end{aligned}
$$

Note that the above can be checked easily in $\rho\left(\Gamma_{0}^{+}(n)\right)$ by GAP.

(ii) Suppose that $n \in E$. Then $v_{4}=1$ if and only if $n=2,10,26,34$, and 50. By results of Maclachlan [Maclachlan 81], every $\sigma \in \Gamma_{0}(n)$ of order 2 is a conjugate of the square of $u \in w_{2} \Gamma_{0}(n)$, where $u$ is elliptic of order 4. As a consequence, elements of order 2 of $\Gamma_{0}(n)$ are not elliptic elements. Further, elliptic elements of order 4 of the above groups are given by

$$
\begin{aligned}
& \left(\begin{array}{cc}
0 & -1 / \sqrt{2} \\
\sqrt{2} & -\sqrt{2}
\end{array}\right) \in w_{2} \Gamma_{0}(2), \\
& \left(\begin{array}{cc}
3 \sqrt{2} & -1 / \sqrt{2} \\
25 \sqrt{2} & -4 \sqrt{2}
\end{array}\right) \in w_{2} \Gamma_{0}(10), \\
& \left(\begin{array}{cc}
3 \sqrt{2} & -1 / \sqrt{2} \\
13 \sqrt{2} & -2 \sqrt{2}
\end{array}\right) \in w_{2} \Gamma_{0}(26), \\
& \left(\begin{array}{cc}
6 \sqrt{2} & -1 / \sqrt{2} \\
85 \sqrt{2} & -7 \sqrt{2}
\end{array}\right) \in w_{2} \Gamma_{0}(34), \\
& \left(\begin{array}{cc}
3 \sqrt{2} & -1 / \sqrt{2} \\
25 \sqrt{2} & -4 \sqrt{2}
\end{array}\right) \in w_{2} \Gamma_{0}(50) .
\end{aligned}
$$

(iii) Suppose that $n \in E$. Then $v_{3}=1$ if and only if $n=1,7,13,19,31$, and 49. Elliptic elements of order 3 of the above groups are given by

$$
\begin{aligned}
& \left(\begin{array}{cc}
1 & -1 \\
1 & 0
\end{array}\right),\left(\begin{array}{cc}
-2 & -1 \\
7 & 3
\end{array}\right), \\
& \left(\begin{array}{cc}
-3 & -1 \\
13 & 4
\end{array}\right),\left(\begin{array}{cc}
-7 & -3 \\
19 & 8
\end{array}\right), \\
& \left(\begin{array}{cc}
-5 & -1 \\
31 & 6
\end{array}\right),\left(\begin{array}{cc}
-18 & -7 \\
49 & 19
\end{array}\right) .
\end{aligned}
$$

Denote the matrix

$$
\left(\begin{array}{cc}
x & y \\
z & w
\end{array}\right)
$$

by $(x, y: z, w)$. Table 6 gives a complete set of representatives of nonconjugate elliptic subgroups of order 2 of $\Gamma_{0}^{+}(n)$, where $n \in E$ and $g\left(\Gamma_{0}^{+}(n)\right)=0$.

\section{THE CUSPS OF $\Gamma_{0}(N)$ AND $\Gamma(N)$}

The purpose of this section is to study the cusps of $\Gamma_{0}(N)$ and $\Gamma(N)$. Let $N \in \mathbb{N}$ and let $h$ be the largest divisor of 24 such that $N=n h^{2}$. The following gives a complete description of the set of cusps of $\Gamma_{0}\left(n h^{2}\right)$ and a systematic description of $\rho(\tau)$, where $\tau \in \Gamma_{0}(n h \mid h)$.

Let $d$ be a positive divisor of $N$ and let $e=$ $\operatorname{gcd}(d, N / d)$. Denote by $S_{d}$ the following set:

$$
\begin{aligned}
S_{d}=\left\{x_{i} / d: \operatorname{gcd}\left(x_{i}, d\right)=1,0 \leq\right. & x_{i} \leq d-1, \\
& \left.x_{i} \not \equiv x_{j}(\bmod e)\right\} .
\end{aligned}
$$

Then $\Delta=\cup_{d \mid N} S_{d}$ is a complete set of inequivalent cusps of $\Gamma_{0}(N)$. Note that $\infty$ is equivalent to $1 / N$.

Remark 6.1. Let $d$ be a positive divisor of $N$ and let $e=$ $\operatorname{gcd}(d, N / d)$. Suppose that $\operatorname{gcd}(x, d)=1=\operatorname{gcd}(y, d)$. Then $x / d$ and $y / d$ are equivalent to each other if and only if $x \equiv y(\bmod e)$.

Let $\tau \in \Gamma_{0}^{+}(n h \mid h)$. The permutation $\rho(\tau)$ can be determined easily by the following lemma.

Lemma 6.2. Let $x / n_{0} y$ be a cusp of $\Gamma_{0}(n)$, where $n_{0}$ is a divisor of $n$ and $\operatorname{gcd}\left(n, n_{0} y\right)=n_{0}, \operatorname{gcd}\left(x, n_{0} y\right)=$ 1. Let $r$ be chosen such that $1 \leq r \leq n_{0}, r \equiv$ $x y\left(\bmod \left(n_{0}, n / n_{0}\right)\right), \operatorname{gcd}\left(r, n_{0}\right)=1$. Then $x / n_{0} y$ is equivalent to $r / n_{0}$.

Proof: Let

$$
\sigma=\left(\begin{array}{cc}
a & b \\
c n & d
\end{array}\right) \in \Gamma_{0}(n)
$$

be chosen such that $c n x+d n_{0} y=n_{0}$. This implies that $\sigma\left(x / n_{0} y\right)=\frac{a x+b n_{0} y}{n_{0}}$. Let

$$
\tau=\left(\begin{array}{cc}
1 & -b y \\
0 & 1
\end{array}\right) \in \Gamma_{0}(n) .
$$

It follows that $\tau \sigma\left(x / n_{0} y\right)=a x / n_{0}, \operatorname{gcd}\left(a x, n_{0}\right)=1$. Direct calculation shows that $c n x+d n_{0} y=n_{0}, a d-b c n=$ 1 . Hence $a \equiv y\left(\bmod n / n_{0}\right)$. This implies that $a x / n_{0}=$ $\left(x y+n k / n_{0}\right) / n_{0}$, for some $k \in \mathbb{Z}$. Since $\left(x y+n k / n_{0}\right) / n_{0}$ is equivalent to $s / n_{0}$ if and only if $x y+n k / n_{0} \equiv s(\bmod$ $\left(n_{0}, n / n_{0}\right)$ ) (see Remark 6.1), it follows that $x / n_{0} y$ is equivalent to $r / n_{0}$. 


\begin{tabular}{|c|c|c|}
\hline$n$ & $v_{2}$ & elliptic elements of order 2 \\
\hline 1 & 1 & $(0,-1: 1,0)$ \\
\hline 2 & 1 & $(0,-1 / \sqrt{2}: \sqrt{2}, 0)$ \\
\hline 3 & 1 & $(0,-1 / \sqrt{3}: \sqrt{3}, 0)$ \\
\hline 4 & 1 & $(0,-1 / 2: 2,0)$ \\
\hline 5 & 3 & $(3,-2: 5,-3),(0,-1 / \sqrt{5}: \sqrt{5}, 0),(\sqrt{5},-3 / \sqrt{5}: 2 \sqrt{5},-\sqrt{5})$ \\
\hline 6 & 3 & $(\sqrt{2},-1 / \sqrt{2}: 3 \sqrt{2},-\sqrt{2}),(\sqrt{3},-1 / \sqrt{3}: 4 \sqrt{3},-\sqrt{3}),(0,-1 / \sqrt{6}: \sqrt{6}, 0)$ \\
\hline 7 & 2 & $(0,-1 / \sqrt{7}: \sqrt{7}, 0),(\sqrt{7},-2 / \sqrt{7}: 4 \sqrt{7},-\sqrt{7})$ \\
\hline 8 & 2 & $(0,-1 / \sqrt{8}: \sqrt{8}, 0),(\sqrt{8},-3 / \sqrt{8}: 3 \sqrt{8},-\sqrt{8})$ \\
\hline 9 & 2 & $(0,-1 / \sqrt{9}: \sqrt{9}, 0),(\sqrt{9},-5 / \sqrt{9}: 2 \sqrt{9},-\sqrt{9})$ \\
\hline 10 & 2 & $(\sqrt{5},-3 / \sqrt{5}: 2 \sqrt{5},-\sqrt{5}),(0,-1 / \sqrt{10}: \sqrt{10}, 0)$ \\
\hline 11 & 4 & $\begin{array}{l}(0,-1 / \sqrt{11}: \sqrt{11}, 0),(\sqrt{11},-2 / \sqrt{11}: 6 \sqrt{11},-\sqrt{11}),(\sqrt{11},-4 / \sqrt{11}: 3 \sqrt{11},-\sqrt{11}) \\
(\sqrt{11},-3 / \sqrt{11}: 4 \sqrt{11},-\sqrt{11})\end{array}$ \\
\hline 12 & 2 & $(0,-1 / \sqrt{12}: \sqrt{12}, 0),(\sqrt{3},-1 / \sqrt{3}: 4 \sqrt{3},-\sqrt{3})$ \\
\hline 13 & 3 & $(5,-2: 13,-5),(0,-1 / \sqrt{13}: \sqrt{13}, 0),(\sqrt{13},-2 / \sqrt{13}: 7 \sqrt{13},-\sqrt{13})$ \\
\hline 14 & 4 & $\begin{array}{l}(\sqrt{7},-1 / \sqrt{7}: 8 \sqrt{7},-\sqrt{7}),(\sqrt{7},-2 / \sqrt{7}: 4 \sqrt{7},-\sqrt{7}),(0,-1 / \sqrt{14}: \sqrt{14}, 0) \\
(\sqrt{14},-3 / \sqrt{14}: 5 \sqrt{14},-\sqrt{14})\end{array}$ \\
\hline 15 & 4 & $\begin{array}{l}(\sqrt{5},-2 / \sqrt{5}: 3 \sqrt{5},-\sqrt{5}),(\sqrt{5},-1 / \sqrt{5}: 6 \sqrt{5},-\sqrt{5}),(0,-1 / \sqrt{15}: \sqrt{15}, 0) \\
(\sqrt{15},-2 / \sqrt{15}: 8 \sqrt{15},-\sqrt{15})\end{array}$ \\
\hline 16 & 2 & $(0,-1 / \sqrt{16}: \sqrt{16}, 0),(2 \sqrt{16},-13 / \sqrt{16}: 5 \sqrt{16},-2 \sqrt{16})$ \\
\hline 17 & 5 & $\begin{array}{l}(4,-1: 17,-4),(0,-1 / \sqrt{17}: \sqrt{17}, 0),(\sqrt{17},-2 / \sqrt{17}: 9 \sqrt{17},-\sqrt{17}) \\
(\sqrt{17},-6 / \sqrt{17}: 3 \sqrt{17},-\sqrt{17}),(\sqrt{17},-3 / \sqrt{17}: 6 \sqrt{17},-\sqrt{17})\end{array}$ \\
\hline 18 & 3 & $(0,-1 / \sqrt{18}: \sqrt{18}, 0),(\sqrt{9},-5 / \sqrt{9}: 2 \sqrt{9},-\sqrt{9}),(2 \sqrt{2},-1 / \sqrt{2}: 9 \sqrt{2},-2 \sqrt{2})$ \\
\hline 19 & 4 & $\begin{array}{l}(0,-1 / \sqrt{19}: \sqrt{19}, 0),(\sqrt{19},-2 / \sqrt{19}: 10 \sqrt{19},-\sqrt{19}),(\sqrt{19},-5 / \sqrt{19}: 4 \sqrt{19},-\sqrt{19}) \\
(\sqrt{19},-4 / \sqrt{19}: 5 \sqrt{19},-\sqrt{19})\end{array}$ \\
\hline 21 & 3 & $(4 \sqrt{3},-7 / \sqrt{3}: 7 \sqrt{3},-4 \sqrt{3}),(0,-1 / \sqrt{21}: \sqrt{21}, 0),(\sqrt{21},-2 / \sqrt{21}: 11 \sqrt{21},-\sqrt{21})$ \\
\hline 22 & 5 & $\begin{array}{l}(4 \sqrt{2},-3 / \sqrt{2}: 11 \sqrt{2},-4 \sqrt{2}),(\sqrt{11},-1 / \sqrt{11}: 12 \sqrt{11},-\sqrt{11}),(\sqrt{11},-3 / \sqrt{11}: 4 \sqrt{11},-\sqrt{11}) \\
(\sqrt{11},-2 / \sqrt{11}: 6 \sqrt{11},-\sqrt{11}),(0,-1 / \sqrt{22}: \sqrt{22}, 0)\end{array}$ \\
\hline 23 & 6 & $\begin{array}{l}(0,-1 / \sqrt{23}: \sqrt{23}, 0),(\sqrt{23},-2 / \sqrt{23}: 12 \sqrt{23},-\sqrt{23}),(\sqrt{23},-3 / \sqrt{23}: 8 \sqrt{23},-\sqrt{23}) \\
\sqrt{23},-4 / \sqrt{23}: 6 \sqrt{23},-\sqrt{23}),(\sqrt{23},-6 / \sqrt{23}: 4 \sqrt{23},-\sqrt{23}),(\sqrt{23},-8 / \sqrt{23}: 3 \sqrt{23},-\sqrt{23})\end{array}$ \\
\hline 24 & 4 & $\begin{array}{l}([0,-1 / \sqrt{24}: \sqrt{24}, 0),(\sqrt{8},-3 / \sqrt{8}: 3 \sqrt{8},-\sqrt{8}),(\sqrt{8},-1 / \sqrt{8} ; 9 \sqrt{8},-\sqrt{8}) \\
(\sqrt{24},-5 / \sqrt{24} ; 5 \sqrt{24},-\sqrt{24})\end{array}$ \\
\hline 25 & 3 & $(0,-1 / \sqrt{25}: \sqrt{25}, 0)(\sqrt{25},-13 / \sqrt{25}: 2 \sqrt{25},-\sqrt{25}),(7,-2: 25,-7)$ \\
\hline 26 & 4 & $\begin{array}{l}(\sqrt{13},-7 / \sqrt{13}: 2 \sqrt{13},-\sqrt{13}),(0,-1 / \sqrt{26}: \sqrt{26}, 0),(\sqrt{26},-3 / \sqrt{26}: 9 \sqrt{26},-\sqrt{26}) \\
(\sqrt{26},-9 / \sqrt{26}: 3 \sqrt{26},-\sqrt{26})\end{array}$ \\
\hline 27 & 4 & $\begin{array}{l}(0,-1 / \sqrt{27}: \sqrt{27}, 0),(\sqrt{27},-14 / \sqrt{27}: 2 \sqrt{27},-\sqrt{27}),(\sqrt{27},-7 / \sqrt{27}: 4 \sqrt{27},-\sqrt{27}) \\
(\sqrt{27},-4 / \sqrt{27}: 7 \sqrt{27},-\sqrt{27})\end{array}$ \\
\hline 29 & 7 & $\begin{array}{l}(12,-5: 29,-12),(0,-1 / \sqrt{29}: \sqrt{29}, 0),(\sqrt{29},-2 / \sqrt{29}: 15 \sqrt{29},-\sqrt{29}) \\
(\sqrt{29},-3 / \sqrt{29}: 10 \sqrt{29},-\sqrt{29}),(\sqrt{29},-5 / \sqrt{29}: 6 \sqrt{29},-\sqrt{29}),(\sqrt{29},-6 / \sqrt{29}: 5 \sqrt{29},-\sqrt{29}), \\
(\sqrt{29},-10 / \sqrt{29}: 3 \sqrt{29},-\sqrt{29})\end{array}$ \\
\hline 30 & 5 & $\begin{array}{l}(\sqrt{5},-1 / \sqrt{5}: 6 \sqrt{5},-\sqrt{5}),(0,-1 / \sqrt{30}: \sqrt{30}, 0),(\sqrt{15},-1 / \sqrt{15}: 16 \sqrt{15},-\sqrt{15}) \\
(\sqrt{15},-2 / \sqrt{15}: 8 \sqrt{15},-\sqrt{15}),(2 \sqrt{6},-5 / \sqrt{6}: 5 \sqrt{6},-2 \sqrt{6})\end{array}$ \\
\hline
\end{tabular}

TABLE 6. 


\begin{tabular}{|c|c|c|}
\hline 31 & 6 & $\begin{array}{l}(\sqrt{31},-2 / \sqrt{31}: 16 \sqrt{31},-\sqrt{31}),(\sqrt{31},-8 / \sqrt{31}: 4 \sqrt{31},-\sqrt{31}),(\sqrt{31},-4 / \sqrt{31}: 8 \sqrt{31},-\sqrt{31}) \\
(0,-1 / \sqrt{31}: \sqrt{31}, 0),(2 \sqrt{31},-25 / \sqrt{31}: 5 \sqrt{31},-2 \sqrt{31}),(2 \sqrt{31},-5 / \sqrt{31}: 25 \sqrt{31},-2 \sqrt{31})\end{array}$ \\
\hline 32 & 4 & $\begin{array}{l}(0,-1 / \sqrt{32}: \sqrt{32}, 0),(\sqrt{32},-11 / \sqrt{32}: 3 \sqrt{32},-\sqrt{32}),(\sqrt{32},-3 / \sqrt{32}: 11 \sqrt{32},-\sqrt{32}) \\
(3 \sqrt{32},-17 / \sqrt{32}: 17 \sqrt{32},-3 \sqrt{32})\end{array}$ \\
\hline 33 & 6 & $\begin{array}{l}(\sqrt{11},-4 / \sqrt{11}: 3 \sqrt{11},-\sqrt{11}),(\sqrt{11},-2 / \sqrt{11}: 6 \sqrt{11},-\sqrt{11}),(\sqrt{11},-1 / \sqrt{11}: 12 \sqrt{11},-\sqrt{11}), \\
(2 \sqrt{11},-3 / \sqrt{11}: 15 \sqrt{11},-2 \sqrt{11}),(0,-1 / \sqrt{33}: \sqrt{33}, 0),(\sqrt{33},-2 / \sqrt{33}: 17 \sqrt{33},-\sqrt{33}),\end{array}$ \\
\hline 34 & 5 & $\begin{array}{l}(5 \sqrt{2},-3 / \sqrt{2}: 17 \sqrt{2},-5 \sqrt{2}),(0,-1 / \sqrt{34}: \sqrt{34}, 0),(\sqrt{34},-5 / \sqrt{34}: 7 \sqrt{34},-\sqrt{34}) \\
(\sqrt{17},-1 / \sqrt{17}: 18 \sqrt{17},-\sqrt{17}),(\sqrt{17},-3 / \sqrt{17}: 6 \sqrt{17},-\sqrt{17})\end{array}$ \\
\hline 35 & 6 & $\begin{array}{l}(2 \sqrt{5},-3 / \sqrt{5}: 7 \sqrt{5},-2 \sqrt{5}),(2 \sqrt{5},-1 / \sqrt{5}: 21 \sqrt{5},-2 \sqrt{5}),(0,-1 / \sqrt{35}: \sqrt{35}, 0), \\
(\sqrt{35},-2 / \sqrt{35}: 18 \sqrt{35},-\sqrt{35}),(\sqrt{35},-3 / \sqrt{35}: 12 \sqrt{35},-\sqrt{35}),(\sqrt{35},-9 / \sqrt{35}: 4 \sqrt{35},-\sqrt{35})\end{array}$ \\
\hline 36 & 2 & $(0,-1 / \sqrt{36}: \sqrt{36}, 0),(2 \sqrt{36},-29 / \sqrt{36}: 5 \sqrt{36},-2 \sqrt{36})$ \\
\hline 38 & 7 & $\begin{array}{l}(3 \sqrt{2},-1 / \sqrt{2}: 19 \sqrt{2},-3 \sqrt{2}),(\sqrt{19},-10 / \sqrt{19}: 2 \sqrt{19},-\sqrt{19}),(\sqrt{19},-5 / \sqrt{19}: 4 \sqrt{19},-\sqrt{19}) \\
(\sqrt{19},-2 / \sqrt{19}: 10 \sqrt{19},-\sqrt{19}),(0,-1 / \sqrt{38}: \sqrt{38}, 0),(\sqrt{38},-13 / \sqrt{38}: 3 \sqrt{38},-\sqrt{38}) \\
(\sqrt{38},-3 / \sqrt{38}: 13 \sqrt{38},-\sqrt{38})\end{array}$ \\
\hline 39 & 5 & $\begin{array}{l}(2 \sqrt{3},-1 / \sqrt{3}: 13 \sqrt{3},-2 \sqrt{3}),(0,-1 / \sqrt{39}: \sqrt{39}, 0),(\sqrt{39},-20 / \sqrt{39}: 2 \sqrt{39},-\sqrt{39}) \\
(\sqrt{39},-10 / \sqrt{39}: 4 \sqrt{39},-\sqrt{39}),(\sqrt{39},-8 / \sqrt{39}: 5 \sqrt{39},-\sqrt{39})\end{array}$ \\
\hline 41 & 9 & $\begin{array}{l}(0,-1 / \sqrt{41}: \sqrt{41}, 0),(\sqrt{41},-2 / \sqrt{41}: 21 \sqrt{41},-\sqrt{41}),(\sqrt{41},-14 / \sqrt{41}: 3 \sqrt{41},-\sqrt{41}) \\
(\sqrt{41},-7 / \sqrt{41}: 6 \sqrt{41},-\sqrt{41}),(\sqrt{41},-6 / \sqrt{41}: 7 \sqrt{41},-\sqrt{41}),(\sqrt{41},-3 / \sqrt{41}: 14 \sqrt{41},-\sqrt{41}) \\
(9,-2: 41,-9),(2 \sqrt{41},-33 / \sqrt{41}: 5 \sqrt{41},-2 \sqrt{41}),(2 \sqrt{41},-5 / \sqrt{41}: 33 \sqrt{41},-2 \sqrt{41})\end{array}$ \\
\hline 42 & 6 & $\begin{array}{l}(3 \sqrt{3},-2 / \sqrt{3}: 14 \sqrt{3},-3 \sqrt{3}),(\sqrt{6},-1 / \sqrt{6}: 7 \sqrt{6},-\sqrt{6}),(\sqrt{14},-5 / \sqrt{14}: 3 \sqrt{14},-\sqrt{14}) \\
(\sqrt{14},-1 / \sqrt{14}: 15 \sqrt{14},-\sqrt{14}),(\sqrt{21},-11 / \sqrt{21}: 2 \sqrt{21},-\sqrt{21}),(0,-1 / \sqrt{42}: \sqrt{42}, 0)\end{array}$ \\
\hline 44 & 6 & $\begin{array}{l}(0,-1 / \sqrt{44}: \sqrt{44}, 0),(\sqrt{11},-3 / \sqrt{11}: 4 \sqrt{11},-\sqrt{11}),(\sqrt{11},-1 / \sqrt{11}: 12 \sqrt{11},-\sqrt{11}) \\
(\sqrt{44},-15 / \sqrt{44}: 3 \sqrt{44},-\sqrt{44}),(\sqrt{44},-5 / \sqrt{44}: 9 \sqrt{44},-\sqrt{44}),(3 \sqrt{11},-5 / \sqrt{11}: 20 \sqrt{11},-3 \sqrt{11})\end{array}$ \\
\hline 45 & 6 & $\begin{array}{l}(0,-1 / \sqrt{45}: \sqrt{45}, 0),(\sqrt{9},-2 / \sqrt{9}: 5 \sqrt{9},-\sqrt{9}),(\sqrt{9},-1 / \sqrt{9}: 10 \sqrt{9},-\sqrt{9}) \\
(\sqrt{45},-23 / \sqrt{45}: 2 \sqrt{45},-\sqrt{45}),(4 \sqrt{5},-9 / \sqrt{5}: 9 \sqrt{5},-4 \sqrt{5}),(4 \sqrt{5},-3 / \sqrt{5}: 27 \sqrt{5},-4 \sqrt{5})\end{array}$ \\
\hline 46 & 8 & $\begin{array}{l}(\sqrt{23},-1 / \sqrt{23}: 24 \sqrt{23},-\sqrt{23}),(\sqrt{23},-6 / \sqrt{23}: 4 \sqrt{23},-\sqrt{23}),(\sqrt{23},-4 / \sqrt{23}: 6 \sqrt{23},-\sqrt{23}) \\
(\sqrt{23},-3 / \sqrt{23}: 8 \sqrt{23},-\sqrt{23}),(\sqrt{23},-2 / \sqrt{23}: 12 \sqrt{23},-\sqrt{23}),(3 \sqrt{23},-52 / \sqrt{23}: 4 \sqrt{23},-3 \sqrt{23}) \\
(0,-1 / \sqrt{46}: \sqrt{46}, 0),(2 \sqrt{46},-5 / \sqrt{46}: 37 \sqrt{46},-2 \sqrt{46})\end{array}$ \\
\hline 47 & 10 & $\begin{array}{l}(0,-1 / \sqrt{47}: \sqrt{47}, 0),(\sqrt{47},-x / \sqrt{47}: y \sqrt{47},-\sqrt{47}), 2 \leq y \leq 16, x y=48 \\
(2 \sqrt{47},-21 / \sqrt{47}: 9 \sqrt{47},-2 \sqrt{47}),(2 \sqrt{47},-7 / \sqrt{47}: 27 \sqrt{47},-2 \sqrt{47})\end{array}$ \\
\hline 49 & 4 & $\begin{array}{l}(0,-1 / \sqrt{49}: \sqrt{49}, 0),(\sqrt{49},-25 / \sqrt{49}: 2 \sqrt{49},-\sqrt{49}) \\
(\sqrt{49},-10 / \sqrt{49}: 5 \sqrt{49},-\sqrt{49}),(\sqrt{49},-5 / \sqrt{49}: 10 \sqrt{49},-\sqrt{49})\end{array}$ \\
\hline 50 & 4 & $\begin{array}{l}(0,-1 / \sqrt{50}: \sqrt{50}, 0),(\sqrt{25},-13 / \sqrt{25}: 2 \sqrt{25},-\sqrt{25}) \\
(\sqrt{50},-17 / \sqrt{50}: 3 \sqrt{50},-\sqrt{50}),(\sqrt{50},-3 / \sqrt{50}: 17 \sqrt{50},-\sqrt{50})\end{array}$ \\
\hline 51 & 8 & $\begin{array}{l}(\sqrt{17},-x / \sqrt{17}: 3 y \sqrt{17},-\sqrt{17}), x=1,2,3,6, x y=6 . \\
(0,-1 / \sqrt{51}: \sqrt{51}, 0),(\sqrt{51},-x / \sqrt{51}: y \sqrt{51},-\sqrt{51}), x=2,4,13, x y=52\end{array}$ \\
\hline 54 & 7 & $\begin{array}{l}(0,-1 / \sqrt{54}: \sqrt{54}, 0),(\sqrt{27},-14 / \sqrt{27}: 2 \sqrt{27},-\sqrt{27}),(\sqrt{27},-7 / \sqrt{27}: 4 \sqrt{27},-\sqrt{27}), \\
(\sqrt{27},-2 / \sqrt{27}: 14 \sqrt{27},-\sqrt{27}),(\sqrt{54},-11 / \sqrt{54}: 5 \sqrt{54},-\sqrt{54}),(\sqrt{54},-5 / \sqrt{54}: 11 \sqrt{54},-\sqrt{54}), \\
(11 \sqrt{2},-9 / \sqrt{2}: 27 \sqrt{2},-11 \sqrt{2})\end{array}$ \\
\hline 55 & 8 & $\begin{array}{l}(2 \sqrt{11},-x / \sqrt{11}: 5 y \sqrt{11},-2 \sqrt{11}), x=1,2,9, x y=9,(3 \sqrt{11},-2 / \sqrt{11}: 50 \sqrt{11},-3 \sqrt{11}) \\
(0,-1 / \sqrt{55}: \sqrt{55}, 0),(\sqrt{55},-x / \sqrt{55}: y \sqrt{55},-\sqrt{55}), y=2,4,7, x y=56 .\end{array}$ \\
\hline 59 & 12 & $\begin{array}{l}(0,-1 / \sqrt{59}: \sqrt{59}, 0),(\sqrt{59},-x / \sqrt{59}: y \sqrt{59},-\sqrt{59}), 2 \leq x \leq 20, x y=60 \\
(3 \sqrt{59},-7 / \sqrt{59}: 76 \sqrt{59},-3 \sqrt{59}),(3 \sqrt{59},-76 / \sqrt{59}: 7 \sqrt{59},-3 \sqrt{59})\end{array}$ \\
\hline
\end{tabular}

TABLE 6. Continued. 


\begin{tabular}{|c|c|c|}
\hline 60 & 10 & $\begin{array}{l}(0,-1 / \sqrt{60}: \sqrt{60}, 0),(\sqrt{15},-4 / \sqrt{15}: 4 \sqrt{15},-\sqrt{15}),(\sqrt{15},-2 / \sqrt{15}: 8 \sqrt{15},-\sqrt{15}) \\
(\sqrt{15},-1 / \sqrt{15}: 16 \sqrt{15},-\sqrt{15},(\sqrt{20},-7 / \sqrt{20}: 3 \sqrt{20},-\sqrt{20}),(\sqrt{20},-1 / \sqrt{20}: 21 \sqrt{20},-\sqrt{20}) \\
(\sqrt{31},-x / \sqrt{31}: 2 y \sqrt{31},-\sqrt{31}), x=1,2,4,8, x y=16 . \\
(3 \sqrt{31},-4 / \sqrt{31}: 70 \sqrt{31},-3 \sqrt{31}),(3 \sqrt{31},-20 / \sqrt{31}: 14 \sqrt{31},-3 \sqrt{31}) \\
(0,-1 / \sqrt{62}: \sqrt{62}, 0),(\sqrt{62},-x / \sqrt{62}: y \sqrt{62},-\sqrt{62}), x=3,7,21, x y=63 .\end{array}$ \\
\hline 66 & 8 & $\begin{array}{l}(4 \sqrt{2},-1 / \sqrt{2}: 33 \sqrt{2},-4 \sqrt{2}),(3 \sqrt{6},-5 / \sqrt{6}: 11 \sqrt{6},-3 \sqrt{6}),(\sqrt{33},-17 / \sqrt{33}: 2 \sqrt{33},-\sqrt{33}), \\
(0,-1 / \sqrt{66}: \sqrt{66}, 0),(2 \sqrt{66},-5 / \sqrt{66}: 53 \sqrt{66},-2 \sqrt{66}),(\sqrt{11},-2 / \sqrt{11}: 6 \sqrt{11},-\sqrt{11}) \\
(\sqrt{11},-1 / \sqrt{11}: 12 \sqrt{11},-\sqrt{11}),(5 \sqrt{11},-2 / \sqrt{11}: 23.6 \sqrt{11},-5 \sqrt{11})\end{array}$ \\
\hline 69 & 10 & $\begin{array}{l}(\sqrt{23},-x / \sqrt{23}: 3 y \sqrt{23},-\sqrt{23}), x=1,2,4,8, x y=8 . \\
(4 \sqrt{23},-3 / \sqrt{23}: 123 \sqrt{23},-4 \sqrt{23}),(5 \sqrt{23},-4 / \sqrt{23}: 144 \sqrt{23},-5 \sqrt{23}) \\
(0,-1 / \sqrt{69}: \sqrt{69}, 0),(\sqrt{69},-x / \sqrt{69}: y \sqrt{69},-\sqrt{69}), x=2,5,10, x y=70 .\end{array}$ \\
\hline 70 & 8 & $\begin{array}{l}(5 \sqrt{5},-9 / \sqrt{5}: 14 \sqrt{5},-5 \sqrt{5}),(3 \sqrt{10},-13 / \sqrt{10}: 7 \sqrt{10},-3 \sqrt{10}),(\sqrt{14},-3 / \sqrt{14}: 5 \sqrt{14},-\sqrt{14}) \\
(\sqrt{14},-1 / \sqrt{14}: 15 \sqrt{14},-\sqrt{14}),(\sqrt{35},-1 / \sqrt{35}: 36 \sqrt{35},-\sqrt{35}),(\sqrt{35},-2 / \sqrt{35}: 18 \sqrt{35},-\sqrt{35}) \\
(\sqrt{35},-6 / \sqrt{35}: 6 \sqrt{35},-\sqrt{35}),(0,-1 / \sqrt{70}: \sqrt{70}, 0)\end{array}$ \\
\hline 71 & 14 & $\begin{array}{l}(0,-1 / \sqrt{71}: \sqrt{71}, 0),(\sqrt{71},-x / \sqrt{71}: y \sqrt{71},-\sqrt{71}), 2 \leq y \leq 24, x y=72 \\
(2 \sqrt{71},-x / \sqrt{71}: y \sqrt{71},-2 \sqrt{71}), x=5,57, x y=285 \\
(3 \sqrt{71},-x / \sqrt{71}: y \sqrt{71},-3 \sqrt{71}), x=8,64, x y=640\end{array}$ \\
\hline 78 & 9 & $\begin{array}{l}(11 \sqrt{3},-1 / \sqrt{26}: 14.29 \sqrt{3},-11 \sqrt{3}),(\sqrt{26},-x / \sqrt{26}: 3 y \sqrt{26},-\sqrt{26}), x=1,3,9, x y=9 . \\
(\sqrt{39},-x / \sqrt{39}: 2 y \sqrt{39},-\sqrt{39}), x=1,2,4,10, x y=20 . \\
(0,-1 / \sqrt{78}: \sqrt{78}, 0)\end{array}$ \\
\hline 87 & 12 & $\begin{array}{l}(\sqrt{29},-x / \sqrt{29}: 3 y \sqrt{29},-\sqrt{29}), x=1,2,5,10, x y=10 . \\
(2 \sqrt{29},-13 / \sqrt{29}: 9 \sqrt{29},-2 \sqrt{29}),(2 \sqrt{29},-3 / \sqrt{29}: 39 \sqrt{29},-2 \sqrt{29}) \\
(0,-1 / \sqrt{87}: \sqrt{87}, 0),(\sqrt{87},-x / \sqrt{87}: y \sqrt{87},-\sqrt{87}), y=2,4,8,11,22, x y=88 .\end{array}$ \\
\hline 92 & 12 & $\begin{array}{l}(0,-1 / \sqrt{92}: \sqrt{92}, 0),(\sqrt{23},-6 / \sqrt{23}: 4 \sqrt{23},-\sqrt{23}),(\sqrt{23},-3 / \sqrt{23}: 8 \sqrt{23},-\sqrt{23}), \\
(\sqrt{23},-2 / \sqrt{23}: 12 \sqrt{23},-\sqrt{23}),(\sqrt{23},-1 / \sqrt{23}: 24 \sqrt{23},-\sqrt{23}),(\sqrt{92},-31 / \sqrt{92}: 3 \sqrt{92},-\sqrt{92}]), \\
(\sqrt{92},-3 / \sqrt{92}: 31 \sqrt{92},-\sqrt{92}),(3 \sqrt{23},-26 / \sqrt{23}: 8 \sqrt{23},-3 \sqrt{23}),(3 \sqrt{23},-13 / \sqrt{23}: 16 \sqrt{23},-3 \sqrt{23}), \\
(3 \sqrt{23},-4 / \sqrt{23}: 52 \sqrt{23},-3 \sqrt{23}),(3 \sqrt{23},-2 / \sqrt{23}: 104 \sqrt{23},-3 \sqrt{23}),(5 \sqrt{23},-18 / \sqrt{23}: 32 \sqrt{23},-5 \sqrt{23})\end{array}$ \\
\hline 94 & 14 & $\begin{array}{l}(\sqrt{47},-x / \sqrt{47}: 2 y \sqrt{47},-\sqrt{47}), x=1,2,3,4,6,8,12, x y=24 . \\
(5 \sqrt{47},-6 / \sqrt{47}: 196 \sqrt{47},-5 \sqrt{47}),(3 \sqrt{47},-x / \sqrt{47}: 2 y \sqrt{47},-3 \sqrt{47}), x=4,53, x y=212 . \\
(0,-1 / \sqrt{94}: \sqrt{94}, 0),(\sqrt{94},-x / \sqrt{94}: y \sqrt{94},-\sqrt{94}), x=5,19, x y=95 . \\
(3 \sqrt{94},-7 / \sqrt{94}: 121 \sqrt{94},-3 \sqrt{94})\end{array}$ \\
\hline 95 & 12 & $\begin{array}{l}(9 \sqrt{19},-4 / \sqrt{19}: 385 \sqrt{19},-9 \sqrt{19}),(\sqrt{19},-x / \sqrt{19}: 5 y \sqrt{19},-\sqrt{19}), x=1,2,4, x y=4 . \\
(0,-1 / \sqrt{95}: \sqrt{95}, 0),(4 \sqrt{95},-9 / \sqrt{95}: 169 \sqrt{95},-4 \sqrt{95}) \\
(\sqrt{95},-x / \sqrt{95}: y \sqrt{95},-\sqrt{95}), x=2,3,4,8,16,32, x y=96\end{array}$ \\
\hline 105 & 10 & $\begin{array}{l}(2 \sqrt{5},-1 / \sqrt{5}: 21 \sqrt{5},-2 \sqrt{5}),(5 \sqrt{5},-1 / \sqrt{5}: 121 \sqrt{5},-5 \sqrt{5}) \\
(2 \sqrt{21},-17 / \sqrt{21}: 5 \sqrt{21},-2 \sqrt{21}),(2 \sqrt{21},-1 / \sqrt{21}: 85 \sqrt{21},-2 \sqrt{21}) \\
(0,-1 / \sqrt{105}: \sqrt{105}, 0),(\sqrt{105},-53 / \sqrt{105}: 2 \sqrt{105},-\sqrt{105}) \\
(\sqrt{35},-x / \sqrt{35}: 3 y \sqrt{35},-\sqrt{35}), x=1,2,3,12, x y=12\end{array}$ \\
\hline 110 & 11 & $\begin{array}{l}(\sqrt{10},-1 / \sqrt{10}: 11 \sqrt{10},-\sqrt{10}),(3 \sqrt{11},-x / \sqrt{11}: 10 y \sqrt{11},-3 \sqrt{11}), x=1,2,10, x y=10 . \\
(\sqrt{55},-x / \sqrt{55}: 2 y \sqrt{55},-\sqrt{55}), x=1,2,4,14, x y=28 \\
(0,-1 / \sqrt{110}: \sqrt{110}, 0),(\sqrt{110},-x / \sqrt{110}: y \sqrt{110},-\sqrt{110}), x=3,37, x y=111\end{array}$ \\
\hline 119 & 14 & $\begin{array}{l}(3 \sqrt{17},-x / \sqrt{17}: 7 y \sqrt{17},-3 \sqrt{17}), x=1,2,11,22, x y=22 .(0,-1 / \sqrt{119}: \sqrt{119}, 0) \\
(\sqrt{119},-x / \sqrt{119}: y \sqrt{119},-\sqrt{119}), x=2,3,4,5,10,12,15,20,24, x y=120\end{array}$ \\
\hline
\end{tabular}

TABLE 6. Continued. 
Example 6.3. The set of cusps of $\Gamma_{0}(144)$ is

$$
\begin{aligned}
\Delta= & \{0,1 / 2,1 / 3,2 / 3,1 / 4,3 / 4,1 / 6,5 / 6,1 / 8,1 / 9,1 / 12, \\
& 5 / 12,7 / 12,11 / 12,1 / 16,1 / 18,1 / 24,5 / 24,1 / 36,7 / 36, \\
& 1 / 48,5 / 48,1 / 72, \infty\} .
\end{aligned}
$$

Let

$$
\tau=\left(\begin{array}{cc}
1 & 0 \\
12 & 1
\end{array}\right) \in \Gamma_{0}(n)
$$

Then $\tau(5 / 48)$ is equivalent to $7 / 36$.

Proof: Direct calculation shows that $\tau(5 / 48)=5 / 36.3$. By Lemma $6.2,5 / 48$ is equivalent to $(5.3+4 k) / 36$ for some $k$. Since $15 \equiv 7(\bmod 4)$ and $\operatorname{gcd}(36,7)=1$, we conclude that $\tau(5 / 48)$ is equivalent to $7 / 36$.

Remark 6.4. Note that since $15 \equiv 11(\bmod 4)$ and $\operatorname{gcd}(36,11)=1$, we have $\tau(5 / 48)$ is equivalent to $11 / 36$ as well.

The following result is taken from [Shimura 71].

Lemma 6.5. Let $a / b, c / d \in \mathbb{Q} \cup\{\infty\}$. Suppose that $\operatorname{gcd}(a, b)=\operatorname{gcd}(c, d)=1$. Then $a / b$ and $c / d$ are equivalent to each other in $\Gamma(n)$ if and only if

(*) $a \equiv c, b \equiv d(\bmod n)$, or $a \equiv-c, b \equiv-d(\bmod n)$.

Further, $\Gamma(n)$ has $\left[P S L_{2}(\mathbb{Z}): \Gamma(n)\right] / n$ cusps.

Applying Lemma 6.5, one may write down a set of representatives of inequivalent cusps as follows.

1. Cusps of $\boldsymbol{\Gamma}(\mathbf{2 m})$. For each $k(1 \leq k \leq m-1)$, define $A_{k}$ to be the set $A_{k}=\{\hat{x}: 1 \leq \hat{x} \leq$ $2 m, \operatorname{gcd}(\operatorname{gcd}(k, \hat{x}), 2 m))=1\}$. For each $\hat{x} \in A_{k}$, let $x=\hat{x}+2 m y$ be the smallest positive integer such that $\operatorname{gcd}(x, k)=1$. Define $S_{k}$ to be the set $S_{k}=\left\{x / k: \hat{x} \in A_{k}\right\}$. Let

$$
\begin{aligned}
A_{m} & =\{\hat{x}: 1 \leq \hat{x} \leq m, \operatorname{gcd}(\operatorname{gcd}(m, \hat{x}), 2 m))=1\}, \\
A_{2 m} & =\{\hat{x}: 1 \leq \hat{x} \leq m, \operatorname{gcd}(\operatorname{gcd}(2 m, \hat{x}), 2 m))=1\} .
\end{aligned}
$$

Define

(i) $S_{m}=\left\{x / m: \hat{x} \in A_{m}\right\}$, where $x=\hat{x}+$ $2 m y$ is the smallest positive integer such that $\operatorname{gcd}(x, m)=1$,

(ii) $S_{2 m}=\left\{x / 2 m: \hat{x} \in A_{2 m}\right\}$, where $x=\hat{x}+$ $2 m y$ is the smallest positive integer such that $\operatorname{gcd}(x, 2 m)=1$.
Corollary 6.6. $S_{1} \cup S_{2} \cup \cdots \cup S_{m-1} \cup S_{m} \cup S_{2 m}$ is a set of cusps of $\Gamma(2 m)$.

Remark 6.7. In $\Gamma(2 m), 1 / 2 m$ is equivalent to $\infty$ and 0 is equivalent to $2 m$.

Example 6.8. A set of inequivalent cusps of $\Gamma(6)$ is given by

$\{\infty=1 / 0,0,1 / 1,2 / 1,3 / 1,4 / 1,5 / 1,1 / 2,3 / 2,5 / 2$, $1 / 3,2 / 3\}$.

2. Cusps of $\boldsymbol{\Gamma}(\mathbf{2} \boldsymbol{m}+\mathbf{1})$. For each $k(1 \leq k \leq m)$, define $A_{k}$ to be the set $A_{k}=\{\hat{x}: 1 \leq \hat{x} \leq 2 m+$ $1, \operatorname{gcd}(\operatorname{gcd}(k, \hat{x}), 2 m+1))=1\}$. For each $\hat{x} \in A_{k}$, let $x=\hat{x}+(2 m+1) y$ be the smallest positive integer such that $\operatorname{gcd}(x, k)=1$. Define $S_{k}$ to be the set $S_{k}=\left\{x / k: \hat{x} \in A_{k}\right\}$. Let

$\left.A_{2 m+1}=\{\hat{x}: 1 \leq \hat{x} \leq m, \operatorname{gcd}(\operatorname{gcd}(k, \hat{x}), 2 m+1))=1\right\}$.

Define $S_{2 m+1}=\left\{x /(2 m+1): \hat{x} \in A_{2 m+1}\right\}$, where $x=\hat{x}+(2 m+1) y$ is the smallest positive integer such that $\operatorname{gcd}(x, 2 m+1)=1$.

Corollary 6.9. $S_{1} \cup S_{2} \cup \cdots \cup S_{m} \cup S_{2 m+1}$ is a set of cusps of $\Gamma(2 m+1)$.

Remark 6.10. In $\Gamma(2 m+1), 1 /(2 m+1)$ is equivalent to $\infty$ and 0 is equivalent to $2 m+1$.

\section{Example 6.11.}

(i) A set of inequivalent cusps of $\Gamma(5)$ is given by $\{\infty, 0,1 / 1,2 / 1,3 / 1,4 / 1,1 / 2,3 / 2,5 / 2,7 / 2,9 / 2,2 / 5\}$.

(ii) A set of inequivalent cusps of $\Gamma(7)$ is given by $\{\infty, 0,1 / 1,2 / 1,3 / 1,4 / 1,5 / 1,6 / 1,1 / 2,3 / 2,5 / 2,7 / 2$, $9 / 2,11 / 2,13 / 2,1 / 3,2 / 3,10 / 3,4 / 3,5 / 3,13 / 3,7 / 3$, $2 / 7,3 / 7\}$.

Remark 6.12. By Lemma 6.5, the permutation representation of $P S L_{2}(\mathbb{Z})$ on the set of cusps of $\Gamma(n)$ can be determined easily. Recall that

$$
P S L_{2}(\mathbb{Z})=\left\langle\left(\begin{array}{ll}
1 & 1 \\
0 & 1
\end{array}\right),\left(\begin{array}{cc}
0 & 1 \\
-1 & 0
\end{array}\right)\right\rangle .
$$




\section{PROOF}

The main purpose of this section is to give a proof of (i) of Section 2.2. Our proof can be found in Section 7.2.

\subsection{Some Basic Results}

Let $M$ be a maximal discrete subgroup of $P S L_{2}(\mathbb{R})$ commensurable with $P S L_{2}(\mathbb{Z})$. By results of Helling [Helling 66], $M$ is a conjugate of $\Gamma_{0}^{+}(f)$, where $f$ is square-free.

Definition 7.1. Let $\nu$ be an element of order 6 of $M$ and let $G$ be a subgroup of $M$. Suppose that $M=\cup_{i=1}^{n} x_{i} G$. We define the following:

(i) $\Delta_{\nu}(G)=\left\{x_{i}: x_{i}^{-1} \nu x_{i} \in G\right\}$,

(ii) for each $x \in \Delta_{\nu^{2}}(G)-\Delta_{\nu}(G)$, define $E_{x}=\left\{x_{i} \in\right.$ $\Delta_{\nu^{2}}(G)-\Delta_{\nu}(G): x_{i}^{-1} \nu^{2} x_{i}$ and $x^{-1} \nu^{2} x$ are conjugate to each other in $G$ \},

(iii) for each $x \in \Delta_{\nu^{3}}(G)-\Delta_{\nu}(G)$, define $F_{x}=\left\{x_{i} \in\right.$ $\Delta_{\nu^{3}}(G)-\Delta_{\nu}(G): x_{i}^{-1} \nu^{3} x_{i}$ and $x^{-1} \nu^{3} x$ are conjugate to each other in $G$ \}.

Remark 7.2. Note that $x^{-1} \nu^{2} x\left(x \in \Delta_{\nu^{2}}(G)-\Delta_{\nu}(G)\right)$ is an elliptic element of order 3 of $G$ (see [Shimura 71, 1.15 and 1.16]). Similarly, $x^{-1} \nu^{3} x\left(x \in \Delta_{\nu^{3}}(G)-\Delta_{\nu}(G)\right)$ is an elliptic element of order 2 of $G$.

The following lemma is taken from Section 4 of [Lang 01]. For readers' convenience, the proof is included.

Lemma 7.3. Let $\nu$ be an elliptic element of order 6 of $M=\cup_{i=1}^{n} x_{i} G$. Then

(i) for any $x, y \in \Delta_{\nu}(G), x^{-1} \nu x$ and $y^{-1} \nu y$ are not conjugate to each other in $G$ if and only if $x \neq y$,

(ii) $\left|E_{x}\right|=2$ for every $x \in \Delta_{\nu}(G)$,

(iii) $\left|F_{x}\right|=3$ for every $x \in \Delta_{\nu}(G)$.

Proof: (i) Suppose that $o(\nu)=6$ and that $\tau_{1}=x_{1}^{-1} \nu x_{1}$, $\tau_{2}=x_{2}^{-1} \nu x_{2} \in G$ are conjugate to each other in $G$. It follows that there exists some $z \in G$ such that $z \tau_{1} z^{-1}=$ $\tau_{2}$. This implies that $x_{2} z x_{1}^{-1}$ commutes with $\nu$. By a standard result of discrete groups (see 1.15 and 1.16 of [Sh]), $x_{2} z x_{1}^{-1}=\nu^{k}$ for some $k$. Hence $z=x_{2}^{-1} \nu^{k} x_{1}=$ $x_{2}^{-1} x_{1} x_{1}^{-1} \nu^{k} x_{1}$. Since $z, x_{1}^{-1} \nu^{k} x_{1} \in G$, we conclude that $x_{2}^{-1} x_{1} \in G$. This implies that $x_{1}=x_{2}$. The rest follows similarly. (iia) For each $x \in \Delta_{\nu^{2}}(G)-\Delta_{\nu}(G)$, there exists a unique $x_{j}$ such that $\nu x G=x_{j} G$. This implies that $x_{j}=\nu x z$ for some $z \in G$. Consequently,

(i) $x_{j}^{-1} \nu^{2} x_{j} \in G$,

(ii) $x_{j}^{-1} \nu x_{j} \notin G\left(x \notin \Delta_{\nu}(G), x^{-1} \nu x \notin G\right)$,

(iii) $x_{j}^{-1} \nu^{2} x_{j}$ and $x^{-1} \nu^{2} x$ are conjugate to each other in $G$.

This implies that $x_{j} \in E_{x}$. Since

(i) $\left(x_{j}^{-1} x\right) x^{-1} \nu x=x_{j}^{-1} \nu x=z^{-1} \in G$,

(ii) $x^{-1} \nu x \notin G$,

we conclude that $x_{j} \neq x$. Hence $\left|E_{x}\right| \geq 2$.

(iib) Suppose that $x_{j} \in E_{x}-\{x\}$. It follows that $x^{-1} \nu^{2} x=z^{-1} x_{j}^{-1} \nu^{2} x_{j} z$ for some $z$ in $G$. Hence $x_{j} z x^{-1}$ commutes with $\nu^{2}$. Consequently, $x_{j} z x^{-1}=\nu^{k}, 0 \leq k \leq$ 5 (see [Shimura 71, 1.15 and 1.16]). This gives

$$
z=x_{j}^{-1} \nu^{k} x=\left(x_{j}^{-1} x\right)\left(x^{-1} \nu^{k} x\right) \in G .
$$

Since

$$
x_{j}^{-1} x \notin G, x^{-1} \nu^{2} x \in G,
$$

$k$ cannot be 0,2 , or 4 . It follows that $x_{j} G=x_{j} z G=$ $\nu^{k} x G, k=1$ or 5 . Since $x^{-1} \nu^{2} x \in G$, we have

$$
x_{j} G=\nu^{k} x G=\nu x G .
$$

It is clear that the choice for such $x_{j}$ is unique. As a consequence, $\left|E_{x}\right| \leq 2$. This completes the proof of (ii). (iii) can be proved similarly.

Proof of (ii) of Section 2.2:

(a) $v_{6}(G)=r$. Suppose that $\left\langle g_{i}^{-1} u g_{i}\right\rangle,\left\langle g_{j}^{-1} u g_{j}\right\rangle \in G$ are conjugate to each other in $G$. Then there exists some $z \in G$ such that $g_{i}^{-1} u g_{i}=z^{-1} g_{j}^{-1} u^{k} g_{j} z$, where $k=1$ or -1 . It follows that

$$
g_{j} z g_{i}^{-1} u g_{i} z^{-1} g_{j}^{-1}=u^{k} .
$$

Since $u$ and $u^{-1}$ are not conjugate to each other (see [Shimura 71, 1.22]), we conclude that

$$
g_{j} z g_{i}^{-1} u g_{i} z^{-1} g_{j}^{-1}=u .
$$

It follows that $g_{i}^{-1} u g_{i}$ and $g_{j}^{-1} u g_{j}$ are conjugate to each other in $G$. Applying (i) of Lemma 7.3, $i=j$. Hence $v_{6}(G)=r$. 
(b) $v_{3}(G)=(k-r) / 2$. Note first that $\left\langle g_{i}^{-1} u^{2} g_{i}\right\rangle$ is an elliptic subgroup of order 3 of $G$ if and only if $g_{i} \in \Delta_{u^{2}}(G)-\Delta_{u}(G)$. Suppose that $\left\langle g_{i}^{-1} u^{2} g_{i}\right\rangle$ and $\left\langle g_{j}^{-1} u^{2} g_{j}\right\rangle$ are conjugate to each other in $G$. Similar to the proof of (a), we conclude that $g_{i}^{-1} u^{2} g_{i}$ and $g_{j}^{-1} u^{2} g_{j}$ are conjugate to each other in $G$. As a consequence, $g_{j} \in E_{g_{i}}$. Applying (ii) of Lemma 7.3, we have $v_{3}(G)=(k-r) / 2$.

Similar to the above, we may show that $v_{2}(G)=e_{1}+$ $e_{2}+\cdots+e_{s}+(e-r) / 3$.

\section{REFERENCES}

[Atkin and Lehner 70] A. O. L. Atkin and J. Lehner. "Hecke Operators on $\Gamma_{0}(m)$." Math. Ann. 185 (1970), 134-160.

[Akbas and Singerman 90] M. Akbas and D. Singerman. "The Normalizer of $\Gamma_{0}(N)$ is $P S L_{2}(\mathbb{R})$." Glasgow Math. J. 32 (1990), 317-327.

[Akbas and Singerman 92] M. Akbas and D. Singerman. "The Signature of the Normalizer of $\Gamma_{0}(N)$." London Mathematical Society Lecture Notes Series 165 (1992), $77-86$.

[Conway 96] J. H. Conway. "Understanding Groups Like $\Gamma_{0}(N) . "$ In Groups, Difference Sets and the Monster, pp. 327-343, Ohio State Univ. Math. Res. Inst. Publ. 4. Berlin: de Gruyter, 1996.

[Conway 79] J. H. Conway and S. P. Norton. "Monstrous Moonshine." Bull. London Math. Soc. 11 (1979), 308338.

[Cummins 04] C. Cummins. "Congruence Subgroups of Groups Commensurable with $P S L_{2}(\mathbb{Z})$ of Genus 0 and 1." Exp. Math. 13:3 (2004), 361-382.
[Cummins and Pauli 03] C. Cummins and S. Pauli. "Congruence Subgroups of $P S L_{2}(\mathbb{Z})$ of Genus Less than or Equal to 24." Exp. Math. 12:2 (2003), 243-255.

[Ferenbaugh 93] C. R. Ferenbaugh. "The Genus-Zero Problem for $n \mid h$-Type Groups." Duke Math. J. 72:1 (1993), $31-63$.

[Harada 87] K. Harada. Modular Functions, Modular Forms and Finite Groups, Lecture Notes at The Ohio State University, 1987.

[Helling 66] H. Helling. "Bestimmung der Kommensurabilitäsklasse der Hilbertschen Moulgruppe." Math. Z. 92 (1966), 269-280.

[Kluit 79] P. G. Kluit. "Hecke Operators on $\Gamma^{*}(N)$ and their Trace." PhD diss., Vrije Universiteit, Amsterdam, 1979.

[Lang 01] M. L. Lang. "The Signature of $\Gamma_{0}^{+}(n) . " J$. of $A l$ gebra 241 (2001), 146-185.

[Maclachlan 81] C. Maclachlan. "Groups of Units of Zero Ternary Quadratic Forms." Proc. Roy. Soc. Edinburgh 88A (1981), 141-157.

[Sebbar 01] A. Sebbar. "Torsion-Free Genus Zero Congruence Subgroups of $P S L_{2}(\mathbb{R})$." Duke Math. J. 110:2 (2001), 376-396.

[Shimura 71] G. Shimura. Introduction to the Arithmetic Theory of Automorphic Functions. Tokyo: Iwanami Shoten, Publishers and Princeton, NJ: Princeton University Press, 1971.

[Thompson 80] J. G. Thompson. "A Finiteness Theorem for Subgroups of $P S L_{2}(\mathbb{R})$ which Are Commensurable with $P_{S L}(\mathbb{Z})$." Proc. Symp. Pure Math. AMS 37 (1980), $533-555$.

[Zograf 91] P. Zograf. "A Spectral Proof of Rademacher's Conjecture for Congruence Subgroups of the Modular Group." J. reine angew. Math. 414 (1991), 113-116.

Kok Seng Chua, Department of Mathematics, National University of Singapore, Singapore, 117543, Republic of Singapore (matlml@math.nus.edu.sg)

Mong Lung Lang, Department of Mathematics, National University of Singapore, Singapore, 117543, Republic of Singapore (matlml@math.nus.edu.sg)

Received August 12, 2003; accepted in revised form March 19, 2004. 